\title{
Simple synthesis of a new family of 22- to 28-membered macrocycles containing two chalcone moieties
}

\author{
Rina Mondal, Tapas K. Mandal, and Asok K. Mallik* \\ Department of Chemistry, Jadavpur University, Kolkata-700 032, India \\ E-mail: mallikak52@yahoo.co.in
}

DOI: $\underline{\text { http://dx.doi.org/10.3998/ark.5550190.0013.909 }}$

\begin{abstract}
A new family of 22- to 28-membered tetrabenzo-macrocycles containing two chalcone moieties has been synthesized from 2-hydroxybenzaldehyde and 2'-hydroxyacetophenone through phenol alkylation with $\alpha, \omega$-dibromoalkanes followed by Claisen-Schmidt reaction under high dilution condition. All the compounds of this family were found to possess both the double bonds in $E$ configuration. An attempt to synthesize another family of isomeric tetrabenzo-macrocycles having opposite alignment of the two chalcone moieties by reaction of 2,2'-dihydroxychalcone with $\alpha, \omega$-dibromoalkanes was not successful, a series of 12- to 14-membered dibenzomacrocycles with only one chalcone moiety was obtained here.
\end{abstract}

Keywords: Combinatorial chemistry, macrocycles, bis-chalcones, Claisen-Schmidt reaction, oxygen heterocycles

\section{Introduction}

The chalcone (1,3-diphenyl-2-propen-1-one) moiety is very easy to construct, and chalcones are reported to possess important biological activities. ${ }^{1-7}$ Moreover, these compounds are known to be transformed into other compounds in a number of ways, many of which are biologically active heterocycles. ${ }^{8-11}$ Macrocycles incorporating more than one chalcone moiety have great potential in generating new compounds suitable for molecular recognition ${ }^{12-15}$ and photophysical $^{16,17}$ studies. The chemical literature shows sporadic reports of construction of a few such types of compounds. ${ }^{18-20}$ Synthesis of other structurally related compounds and their suitable transformations to useful hosts are also reported. ${ }^{21,22}$ Furthermore, very recently several types of macrocycles containing fused heterocyclic or homocyclic moieties have been synthesized $^{23-29}$ and a good number of them have shown some very interesting properties. It was therefore our endeavour to develop a simple synthesis of macrocycles containing more than one chalcone moiety. The study started with synthesis of target molecules containing two chalcone 
moieties by exploiting the well-known Claisen-Schmidt reaction which is one of the most simple and high-yielding organic reactions showing very high atom economy. The outcome of this study is presented herein.

\section{Results and Discussion}

A retrosynthetic analysis of the target macrocycles indicated a very simple approach for their synthesis through the use of commonly available starting materials. It was considered that generation of a bis-aldehyde by joining two benzaldehyde units with a spacer and that of a bisketone by joining two acetophenone units with another spacer followed by their Claisen-Schmidt condensation (first step intermolecular and the second step intramolecular) would lead to the target macrocycles. Hydroxybenzaldehydes and hydroxyacetophenones are readily available compounds, and our study began with the ortho compounds of these two categories. Reaction of $\alpha, \omega$-dibromoalkanes with 2-hydroxybenzaldehyde in 1:2 mole ratio was used to generate the required bis-aldehydes and analogous reaction involving 2'-hydroxyacetophenone was used to generate the required bis-ketones (Scheme 1). $\alpha, \omega$-Dibromoalkanes containing 2-5 methylene units are commercially available compounds, and so we could readily synthesize the bisaldehydes 1a-d and the bis-ketones $\mathbf{2 a - d} .^{30-32}$

2<smiles>O=Cc1ccccc1O</smiles><smiles>BrCCCCBr</smiles>

2<smiles>CC(=O)c1ccccc1O</smiles>

$\underset{\text { Reflux, } 7 \mathrm{~h} .}{\stackrel{\mathrm{KOH}, \mathrm{MeOH}}{\longrightarrow}}$

$\mathrm{K}_{2} \mathrm{CO}_{3}$,

Dry Acetone

Reflux, $12 \mathrm{~h}$.<smiles>O=Cc1ccccc1OCCCCCOc1ccccc1C=O</smiles>

1

a: $m=0 ; \mathbf{b}: m=1 ; \mathbf{c}: m=2 ; \mathbf{d}: m=3$<smiles>CC(=O)c1ccccc1OCCCCC(=O)Oc1ccccc1C(C)=O</smiles>

2

a: $n=0 ; \mathbf{b}: n=1 ; \mathbf{c}: n=2 ; \mathbf{d}: n=3$

Scheme 1. Synthesis of bis-aldehydes and bis-ketones.

For synthesis of macrocyclic bis-chalcones utilizing the above bis-aldehydes and bis-ketones, reactions of sixteen possible combinations of reactants were performed in aqueous methanolic $\mathrm{KOH}$ (Scheme 2), and all of them proved to be successful. An initial study with four examples $(\mathbf{1 a}+\mathbf{2 a}, \mathbf{1 b}+\mathbf{2 b}, \mathbf{1 c}+\mathbf{2 c}, \mathbf{1 d}+\mathbf{2 d})$ showed that application of high dilution technique increased the yield of the macrocycles significantly. ${ }^{33}$ So the high dilution technique was applied for all the combinations (concentration of each of the two components was in the range 1.5 to $1.9 \times 10^{-3}$ $\mathrm{mol} / \mathrm{L}$ ). Under this condition the yields of the macrocycles were moderate to good, though they varied widely (Table 1). The configurations of the double bonds of the chalcone moieties present 
in each macrocyclic system so obtained have been found to be $E$ from characteristic coupling constant values in ${ }^{1} \mathrm{H}$ NMR spectra $\left(J_{\alpha, \beta} \sim 16 \mathrm{~Hz}\right)$. This structural feature was evident also from an X-ray crystallographic study of one of the members (3f, Figure 1). It may be mentioned here that under the applied reaction conditions intramolecular aldol condensation of $\mathbf{2}$ did not take place at all.

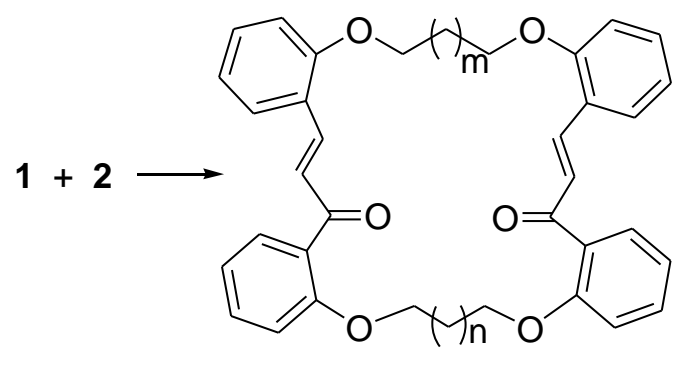

3
a: $m=0, n=0 ; \mathbf{b}: m=0, n=1 ; \mathbf{c}: m=0, n=2 ; d: m=0, n=3$
e: $m=1, n=0 ; \mathbf{f}: m=1, n=1 ; \quad g: m=1, n=2 ; h: m=1, n=3$
i: $m=2, n=0 ; \quad j: m=2, n=1 ; \quad k: m=2, n=2 ; \quad l: m=2, n=3$
$\mathbf{m}: m=3, n=0 ; \mathbf{n}: m=3, n=1 ; \quad \mathbf{o}: m=3, n=2 ; \mathbf{p}: m=3, n=3$

Scheme 2. Synthesis of macrocyclic bis-chalcones from $\mathbf{1}$ and 2.

Table 1. Macrocyclic bis-chalcones synthesized from $\mathbf{1}$ and $\mathbf{2}$

\begin{tabular}{ccccc}
\hline Bis-aldehyde & Bis-ketone & Product & Ring size & $\begin{array}{c}\text { Isolated yield } \\
(\%)\end{array}$ \\
\hline 1a & $\mathbf{2 a}$ & $\mathbf{3 a}$ & 22 & 64 \\
$\mathbf{1 a}$ & $\mathbf{2 b}$ & $\mathbf{3 b}$ & 23 & 72 \\
$\mathbf{1 a}$ & $\mathbf{2 c}$ & $\mathbf{3 c}$ & 24 & 54 \\
$\mathbf{1 a}$ & $\mathbf{2 d}$ & $\mathbf{3 d}$ & 25 & 57 \\
$\mathbf{1 b}$ & $\mathbf{2 a}$ & $\mathbf{3 e}$ & 23 & 70 \\
$\mathbf{1 b}$ & $\mathbf{2 b}$ & $\mathbf{3 f}$ & 24 & 72 \\
$\mathbf{1 b}$ & $\mathbf{2 c}$ & $\mathbf{3 g}$ & 25 & 60 \\
$\mathbf{1 b}$ & $\mathbf{2 d}$ & $\mathbf{3 h}$ & 26 & 58 \\
$\mathbf{1 c}$ & $\mathbf{2 a}$ & $\mathbf{3 i}$ & 24 & 54 \\
$\mathbf{1 c}$ & $\mathbf{2 b}$ & $\mathbf{3 j}$ & 25 & 43 \\
$\mathbf{1 c}$ & $\mathbf{2 c}$ & $\mathbf{3 k}$ & 26 & 56 \\
$\mathbf{1 c}$ & $\mathbf{2 d}$ & $\mathbf{3 1}$ & 27 & 68 \\
$\mathbf{1 d}$ & $\mathbf{2 a}$ & $\mathbf{3 m}$ & 25 & 73 \\
$\mathbf{1 d}$ & $\mathbf{2 b}$ & $\mathbf{3 n}$ & 26 & 62 \\
$\mathbf{1 d}$ & $\mathbf{2 c}$ & $\mathbf{3 0}$ & 27 & 74 \\
$\mathbf{1 d}$ & $\mathbf{2 d}$ & $\mathbf{3 p}$ & 28 & 51 \\
\hline
\end{tabular}




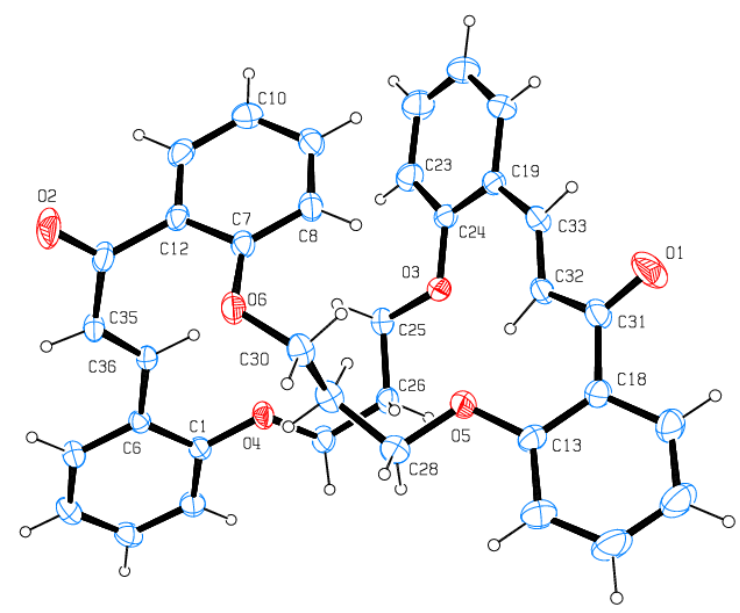

Figure 1. ORTEP diagram of $\mathbf{3 f}$ showing thermal ellipsoids at $25 \%$ probability.

In all the macrocycles 3a-p, the two $\alpha, \beta$-unsaturated ketone moieties are aligned in the same way. There is a possibility of getting the corresponding isomeric macrocycles where these two moieties are aligned in the opposite way, i.e., the macrocycles being of the type 4. Such macrocycles also would be structurally interesting, and for their synthesis etherification involving $\mathbf{5 a}$ and $\mathbf{5 b}$ (through occurrence of an intermolecular process first and then an intramolecular process) was conceived as one of the plausible routes. Anticipating that reaction at the free $\mathrm{OH}$ of 2,2'-dihydroxychalcone (6) would be faster than that at its chelated $\mathrm{OH}$, the mono ethers $\mathbf{5 a}$ and $\mathbf{5 b}$ would be expected to be the products of the first step of its reaction with $\alpha, \omega$-dibromoalkanes. It was our consideration that if it is possible to bring about the second step of the reaction in the same pot, any target compound of the series 4 with $\mathrm{m}=\mathrm{n}$ may be reached readily (Scheme 3). With this synthetic planning, 6 was refluxed separately

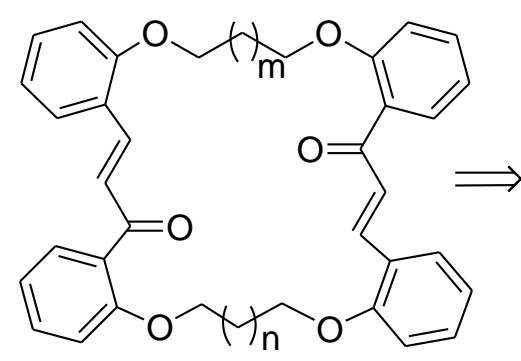

4<smiles>O=C(/C=C/c1ccccc1OCCCOc1ccccc1OCCBr)c1ccccc1OCCBr</smiles>

$5 a$<smiles></smiles>

Scheme 3. Retrosynthetic analysis for construction of 4. 
with each of the $\alpha, \omega$-dibromoalkanes, $\mathrm{Br}-\left(\mathrm{CH}_{2}\right)_{2}-\mathrm{Br}, \mathrm{Br}-\left(\mathrm{CH}_{2}\right)_{3}-\mathrm{Br}, \mathrm{Br}-\left(\mathrm{CH}_{2}\right)_{4}-\mathrm{Br}$ and $\mathrm{Br}-\left(\mathrm{CH}_{2}\right)_{5}-$ $\mathrm{Br}$ in dry acetone with $\mathrm{K}_{2} \mathrm{CO}_{3}$ using the reactants at the concentration $0.067 \mathrm{~mol} / \mathrm{L}$. It was observed that reaction took place in last three of these cases yielding nonphenolic products. However, the spectral data of the products thus obtained showed that, instead of the desired

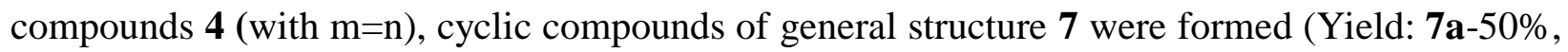
7b-49\%, 7c-54\%) (Scheme 4). Thus, it may be concluded that even by using the reactants in relatively higher concentration as mentioned above, the intramolecular reactions are more facile than intermolecular ones in these cases.
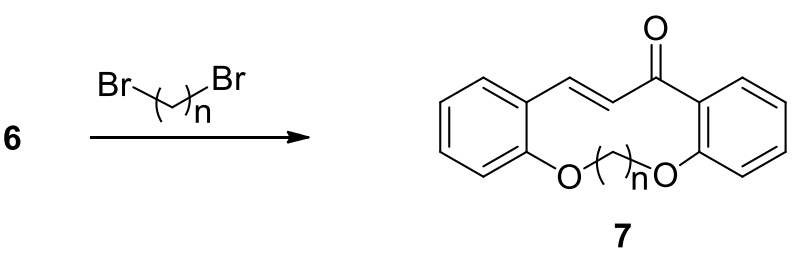

a: $n=3 ; b: n=4 ; c: n=5$

Scheme 4. Alkylation of 6 with $\alpha, \omega$-dibromoalkanes.

A careful examination of the chemical shifts of $\mathrm{H}-\alpha$ and $\mathrm{H}-\beta$ of the enone system of 3a-p showed that the former proton appeared in the range $\delta 7.01-7.55$ while the latter in the range $\delta$ 7.52 - 7.73 (vide Experimental), and the variation of chemical shift values of each of these two protons did not show any regular trend. In case of the compounds $7 \mathbf{a}-\mathbf{c}$, the variation of chemical shifts of $\mathrm{H}-\beta$ and the Ar-H ortho to carbonyl is very conspicuous (Table 2) and here also no regular trend of chemical shift values was observed. The large $\delta$-value for $\mathrm{H}-\beta$ of $\mathbf{7 b}$ indicated that in the preferred conformation of this compound the said proton experiences remarkable anisotropic deshielding.

Table 2. Chemical shift values $(\delta)$ of some protons of 7a-c

\begin{tabular}{cccc}
\hline Compound & H- $\alpha$ & H- $\beta$ & $\begin{array}{c}\text { Ar-H } \\
\text { ortho to } \mathrm{C}=\mathrm{O}\end{array}$ \\
\hline $\mathbf{7 a}$ & 7.52 & 7.60 & 7.72 \\
$\mathbf{7 b}$ & 7.64 & 8.95 & 8.08 \\
$\mathbf{7 c}$ & 7.70 & 8.20 & 7.68 \\
\hline
\end{tabular}

Some initial work towards synthesis of macrocyclic bis-chalcones of the type 8-10 by including 3-hydroxybenzaldehyde and 3'-hydroxyacetophenone also as starting materials could be done so far. Thus, attempts towards synthesis of $\mathbf{8 a}$ (from $\mathbf{1 1}$ and $\mathbf{2 b}$ ), 9a (from 1c and 12) and 10a (from 11 and 12) were found to be successful in the first two cases, though the yields were somewhat lower [8a (ring size 26) - 35\%; 9a (ring size 28) - 38\%]. It is being planned to undertake a separate program for synthesis of these categories of macrocycles. 


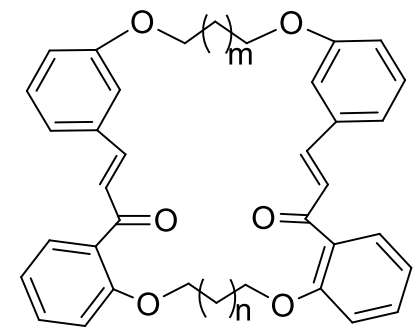

8

(for $8 \mathbf{a}: m=1, n=1$ )

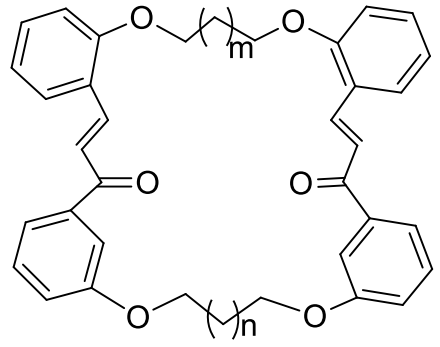

9

(for $9 a: m=2, n=2$ )

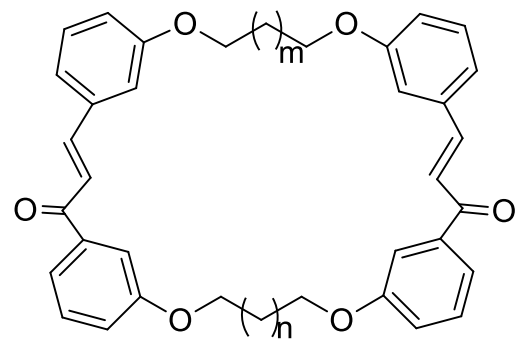

10

(for 10a: $m=1, n=2$ )<smiles>O=Cc1cccc(OCCCOc2cccc(C=O)c2)c1</smiles>

11<smiles>CC(=O)c1cccc(OCC(C)C)c1</smiles>

12

Figure 2. Some possible macrocyclic bis-chalcones starting from 1c, 2b, 11 and 12.

\section{Conclusions}

Thus, a simple method for synthesis of a new family of 22- to 28-membered macrocycles of general structure $\mathbf{3}$, all containing two chalcone moieties with $E$ double bonds, has been developed. As an offshoot of this study, another group of 12- to 14-membered macrocycles (7) containing one $E$ chalcone unit has also been synthesized. The yields of the compounds $\mathbf{3}$ which are of major interest here, have been found to be much better than those for some related compounds reported by other groups. ${ }^{18,19}$ This category of compounds has the potential to find wide applicability as two of their spacers can be varied independently and widely and the two $\alpha, \beta$-unsaturated ketone moieties present in each of them can be made to undergo condensation with varieties of suitable heteroatom containing condensing agents leading to interesting heterocycle fused macrocycles. Some works in this direction are being done currently.

\section{Experimental Section}

General. Melting points were recorded on a Köfler block. IR spectra were recorded on a Perkin Elmer FT-IR Spectrometer (Spectrum RX 1) in $\mathrm{KBr}$ pellets. ${ }^{1} \mathrm{H}$ and ${ }^{13} \mathrm{C}$ NMR spectra were recorded in $\mathrm{CDCl}_{3}$ on a Bruker AV-300 (300 MHz) spectrometer. Mass spectra were measured in the following ways: ESIMS(+) [Waters Micromass Q-ToF micro], FAB-MS [Jeol M Station 
JMS.700], DI-EIMS and GCMS [Shimadzu-QP5050A], and GCMS [Q star XL hybrid instrument]. Analytical samples were routinely dried in vacuo at room temperature. Microanalytical data were recorded on four Perkin-Elmer 2400 Series II C, H, N analyzers. Column chromatography was performed with silica gel (100-200 mesh) and TLC with silica gel $\mathrm{G}$ made of SRL Pvt. Ltd. Petroleum ether had the boiling range $60-80{ }^{\circ} \mathrm{C}$. X-ray single crystal data collection for $\mathbf{3 f}$ was performed with a "Bruker SMART" diffractometer (details given at the end of this section).

\section{1,m-Bis(2-/3-formylphenoxy)alkanes 1a-d and 11. General procedure}

A mixture of 2-/3-hydroxybenzaldehyde $(20 \mathrm{mmol})$ and $\alpha, \omega$-dibromoalkane $(10 \mathrm{mmol})$ was refluxed in methanolic $\mathrm{KOH}(5 \%, 25 \mathrm{~mL})$ for $7 \mathrm{~h}$. Removal of methanol by distillation, addition of water followed by extraction with ethyl acetate gave crude alkylation product which was purified by rapid column chromatography followed by crystallization from $\mathrm{CHCl}_{3}$-petroleum ether. They were characterized from their melting points (given below) and ${ }^{1} \mathrm{H}$ NMR spectral features (data given where necessary).

1,2-Bis-(2-formylphenoxy)ethane (1a). Colourless needles, mp 128-129 ${ }^{\circ} \mathrm{C}$ (lit. ${ }^{8 \mathrm{a}} 128-130{ }^{\circ} \mathrm{C}$ ).

1,3-Bis-(2-formylphenoxy)propane (1b). Colourless needles, mp 98-99 ${ }^{\circ} \mathrm{C}$ (lit. ${ }^{8 a} 99-100{ }^{\circ} \mathrm{C}$ ).

1,4-Bis-(2-formylphenoxy)butane (1c). Colourless needles, mp $145-146{ }^{\circ} \mathrm{C}$ (lit. ${ }^{8 \mathrm{a}} 146-148{ }^{\circ} \mathrm{C}$ ).

1,5-Bis-(2-formylphenoxy)pentane (1d). Colourless needles, mp $57^{\circ} \mathrm{C}$ (lit. ${ }^{8 \mathrm{~b}} 50-53{ }^{\circ} \mathrm{C}$ ).

1,3-Bis-(3-formylphenoxy)propane (11). Colourless needles, mp $68{ }^{\circ} \mathrm{C}$; ${ }^{1} \mathrm{H}$ NMR $(300 \mathrm{MHz}$, $\mathrm{CDCl}_{3}$ ): $\delta_{\mathrm{H}} 2.32$ (quintet, $\left.2 \mathrm{H}, J 6 \mathrm{~Hz}\right), 4.24(\mathrm{t}, 4 \mathrm{H}, J 6 \mathrm{~Hz}), 7.17-7.20(\mathrm{~m}, 2 \mathrm{H}), 7.41-7.47(\mathrm{~m}$, $6 \mathrm{H}), 9.97$ (br. s, 2H, $2 \times-\mathrm{CHO}$ ).

\section{1,n-Bis-(2-/3-acetylphenoxy)alkanes 2a-d and 12. General procedure}

A mixture of 2'-/3'-hydroxyacetophenone (20 mmol), $\alpha, \omega$-dibromoalkane $(10 \mathrm{mmol})$ and anhyd. $\mathrm{K}_{2} \mathrm{CO}_{3}$ (3g.) was refluxed in dry acetone for $12 \mathrm{~h}$. Usual work-up followed by purification of the resulting crude material by column chromatography over silica gel afforded pure product. They were characterized from their melting points (given below) and ${ }^{1} \mathrm{H}$ NMR spectral features (data given where necessary).

1,2-Bis-(2-acetylphenoxy)ethane (2a). Colourless needles, mp $126{ }^{\circ} \mathrm{C}$ (lit. $\left.{ }^{8 \mathrm{c}} 125^{\circ} \mathrm{C}\right)$. 1,3-Bis-(2-acetylphenoxy)propane (2b). Colourless needles, mp 104-106 ${ }^{\circ} \mathrm{C}$ (lit. ${ }^{8 \mathrm{c}} 107{ }^{\circ} \mathrm{C}$ ).

1,4-Bis-(2-acetylphenoxy)butane (2c). Colourless needles, mp 148-150 ${ }^{\circ} \mathrm{C} ;{ }^{1} \mathrm{H}$ NMR $(300$ $\mathrm{MHz}, \mathrm{CDCl}_{3}$ ): $\delta 2.07$ (br. s, 4H, w $1 / 2 \approx 13.0 \mathrm{~Hz}$ ), 2.61 (s, 6H, $2 \times-\mathrm{COCH}_{3}$ ), 4.14 (br. s, 4H, $\mathrm{w}_{1 / 2} \approx 13.0 \mathrm{~Hz}$ ), 6.95 (br. d, 2H, J $8.4 \mathrm{~Hz}$ ), 7.00 (br. t, 2H, J 7.6 Hz), 7.45 (ddd, 2H, J 8.4, 7.7 and $1.8 \mathrm{~Hz}), 7.73(\mathrm{dd}, 2 \mathrm{H}, \mathrm{J} 7.7$ and $1.8 \mathrm{~Hz})$.

1,5-Bis-(2-acetylphenoxy)pentane (2d). Colourless needles, mp $106{ }^{\circ} \mathrm{C}$ (lit. ${ }^{8 b} 105-106{ }^{\circ} \mathrm{C}$ ).

1,4-Bis-(3-acetylphenoxy)butane (12). Colourless needles, mp $105{ }^{\circ} \mathrm{C}$; ${ }^{1} \mathrm{H} \mathrm{NMR}(300 \mathrm{MHz}$, $\mathrm{CDCl}_{3}$ ): $\delta 2.01$ (br. s, $4 \mathrm{H}, \mathrm{w}_{1 / 2} \approx 13.0 \mathrm{~Hz}$ ), 2.59 (s, $6 \mathrm{H}, 2 \times-\mathrm{COCH}_{3}$ ), 4.09 (br. s, 4H, w $1 / 2 \approx 13.0$ Hz), 7.10 (br. d, 2H, J 8.0 Hz), 7.36 (t, 2H, J 7.9 Hz), 7.48 (br. s, 2H) and 7.53 (br. d, 2H, J 7.7 $\mathrm{Hz})$. 
General procedure for macrocyclisation using the bis-aldehydes 1 and the bis-ketones 2 . A mixture of bis-aldehyde (1) $(0.25 \mathrm{mmol})$ and bis-ketone (2) $(0.25 \mathrm{mmol})$ was dissolved in a $\mathrm{KOH}$ solution $(10 \%, 130-160 \mathrm{~mL})$ in $\mathrm{MeOH}-\mathrm{H}_{2} \mathrm{O}$ (3:1) and the mixture was stirred at room temperature. A precipitate began to be formed after $c a .5 \mathrm{~h}$. of stirring. The stirring was continued for $72 \mathrm{~h}$., the volume of the solvent was reduced to nearly half of the original by distillation under reduced pressure. The product obtained as precipitate was collected by careful filtration. The solid thus obtained was almost pure and it was further purified by column chromatography over silica gel followed by crystallization from $\mathrm{CHCl}_{3}$-petroleum ether. The analytical and spectral data of the macrocyclic products 3a-p were as follows:

$(2 E, 22 E)-11,14,30,33-T e t r a o x a p e n t a c y c l o\left[32.4 .0 .0^{5,10} \cdot 0^{15,20} \cdot 0^{24,29}\right]$ octatriaconta-1(38),2,5,7,9,$\mathbf{1 5 , 1 7 , 1 9 , 2 2 , 2 4 , 2 6 , 2 8 , 3 4 , 3 6 - t e t r a d e c a e n e - 4 , 2 1 - d i o n e ~ ( 3 a ) . ~ E l u e n t : ~ p e t r o l e u m ~ e t h e r - E t O A c ~ ( 6 : 4 , ~}$ v/v), light yellow cubes, mp 225-227 ${ }^{\circ} \mathrm{C}$; IR (KBr): 1628 (CO), 1597, 1487, 1472, $1455 \mathrm{~cm}^{-1} ;{ }^{1} \mathrm{H}$ NMR (300 MHz, $\left.\mathrm{CDCl}_{3}\right) \delta_{\mathrm{H}}: 4.17\left(\mathrm{~s}, 4 \mathrm{H},-\mathrm{CH}_{2}-\mathrm{CH}_{2}-\right), 4.22\left(\mathrm{~s}, 4 \mathrm{H},-\mathrm{CH}_{2}-\mathrm{CH}_{2}\right), 6.52(\mathrm{~d}, 2 \mathrm{H}, J$ $8.2 \mathrm{~Hz}), 6.81(\mathrm{~d}, 2 \mathrm{H}, J 8.3 \mathrm{~Hz}), 6.88(\mathrm{t}, 2 \mathrm{H}, J 7.4 \mathrm{~Hz}), 6.99(\mathrm{t}, 2 \mathrm{H}, J 7.4 \mathrm{~Hz}), 7.14(\mathrm{t}, 2 \mathrm{H}, J 8.1$ $\mathrm{Hz}), 7.14(\mathrm{~d}, 2 \mathrm{H}, J 16.3 \mathrm{~Hz}, 2 \times \mathrm{H}-\alpha), 7.34$ (t, 2H, $J .5 \mathrm{~Hz}), 7.38$ (dd, 2H, $J 7.8$ and $1.5 \mathrm{~Hz}$ ), $7.57(\mathrm{~d}, 2 \mathrm{H}, J 7.5 \mathrm{~Hz}), 7.59$ (d, 2H, J $16.4 \mathrm{~Hz}, 2 \times \mathrm{H}-\beta) ;{ }^{13} \mathrm{C} \mathrm{NMR}\left(75 \mathrm{MHz}, \mathrm{CDCl}_{3}\right) \delta_{\mathrm{C}}: 66.89$, 67.35, 112.14, 112.65, 120.92, 121.38, 123.96, 128.13, 128.46, 129.44, 129.67, 131.40, 131.97, 139.56, 156.55, 157.32, 195.07 (CO); FABMS: $m / z 533(\mathrm{M}+\mathrm{H})^{+}$; Anal. Calcd for $\mathrm{C}_{34} \mathrm{H}_{28} \mathrm{O}_{6}$ (532.19): C, 76.68; H, 5.30\%. Found C, 76.48; H 5.43\%.

$(2 E, 23 E)-11,15,31,34-T e t r a o x a p e n t a c y c l o\left[33.4 .0 .0^{5,10} \cdot 0^{16,21} \cdot 0^{25,30}\right]$ nonatriaconta-1(39),2,5,7,$\mathbf{9 , 1 6 , 1 8 , 2 0 , 2 3 , 2 5 , 2 7 , 2 9 , 3 5 , 3 7 - t e t r a d e c a e n e - 4 , 2 2 - d i o n e ~ ( 3 b ) . ~ E l u e n t : ~ p e t r o l e u m ~ e t h e r - E t O A c ~}$ $(6: 4, \mathrm{v} / \mathrm{v})$, light yellow cubes, $\mathrm{mp} 227-229^{\circ} \mathrm{C}$; IR (KBr):1633 (CO), 1582, 1486, 1469, $1387 \mathrm{~cm}^{-}$ 1; ${ }^{1} \mathrm{H}$ NMR (300 MHz, $\mathrm{CDCl}_{3}$ ) $\delta_{\mathrm{H}}: 2.00$ (quintet, $2 \mathrm{H}, J 5.1 \mathrm{~Hz},-\mathrm{CH}_{2}-\mathrm{C}_{2}-\mathrm{CH}_{2}$ ), $3.85(\mathrm{t}, 4 \mathrm{H}, J$ $\left.5.1 \mathrm{~Hz},-\underline{\mathrm{C}}_{2}-\mathrm{CH}_{2}-\underline{\mathrm{C}}_{2}-\right), 4.08\left(\mathrm{~s}, 4 \mathrm{H},-\mathrm{CH}_{2}-\mathrm{CH}_{2}-\right), 6.21(\mathrm{~d}, 2 \mathrm{H}, J$ 8.1 Hz), 6.69-6.75 (m, 4H), 6.85 (br. t, 2H, J 7.6 Hz), 7.01 (dd, 2H, J 16.5 and $1 \mathrm{~Hz}, 2 \times \mathrm{H}-\alpha), 7.08$ (t, 2H, J 7.5 Hz), 7.18 (br. d, 2H, J 7.1 Hz), 7.38 (br. t, 2H, J 7.7 Hz), 7.52 (d, 2H, J $16.3 \mathrm{~Hz}, 2 \times \mathrm{H}-\beta$ ), 7.64 (br. d, 2H, $J 7.6 \mathrm{~Hz}) ;{ }^{13} \mathrm{C} \mathrm{NMR}\left(75 \mathrm{MHz}, \mathrm{CDCl}_{3}\right) \delta_{\mathrm{C}}: 28.40,63.94,68.12,112.10,113.65,121.03,121.34$, 124.04, 128.56, 129.56, 130.12, 131.04, 131.21, 132.27, 140.01, 156.70, 157.45, 194.53 (CO); FABMS: $m / z 547(\mathrm{M}+\mathrm{H})^{+}$; Anal. Calcd for $\mathrm{C}_{35} \mathrm{H}_{30} \mathrm{O}_{6}$ (546.61): C, 76.91; H, 5.53\%. Found C, $76.63 ; \mathrm{H}, 5.61 \%$.

$(2 E, 24 E)-11,16,32,35-T e t r a o x a p e n t a c y c l o\left[34.4 .0 .0^{5,10} \cdot 0^{17,22} \cdot 0^{26,31}\right]$ tetraconta-1(40),2,5,7,9,17,19,21,24,26,28,30,36,38-tetradecaene-4,23-dione (3c). Eluent: petroleum ether-EtOAc (1:1, $\mathrm{v} / \mathrm{v})$, light yellow cubes, mp 198-200 ${ }^{\circ} \mathrm{C}$; IR (KBr): 1626 (CO), 1597, 1485, 1447, $1385 \mathrm{~cm}^{-1} ;{ }^{1} \mathrm{H}$ NMR (300 MHz, $\left.\mathrm{CDCl}_{3}\right) \delta_{\mathrm{H}}: 1.68$ (br. s, 4H, w $1 / 2 \approx 12.5 \mathrm{~Hz},-\mathrm{CH}_{2}-\underline{\mathrm{C}}_{2}-\underline{\mathrm{C}}_{2}-\mathrm{CH}_{2}$ ), 3.56 (br. s, $\left.4 \mathrm{H}, \mathrm{w}_{1 / 2} \approx 12.5 \mathrm{~Hz},-\underline{\mathrm{CH}}_{2}-\mathrm{CH}_{2}-\mathrm{CH}_{2}-\underline{\mathrm{C}}_{2}-\right), 4.21\left(\mathrm{~s}, 4 \mathrm{H},-\mathrm{CH}_{2}-\mathrm{CH}_{2}-\right), 6.37$ (d, 2H, J 8.4 Hz), 6.85 $(\mathrm{d}, 2 \mathrm{H}, J 8.4 \mathrm{~Hz}), 6.95(\mathrm{t}, 2 \mathrm{H}, J 7.5 \mathrm{~Hz}), 7.04(\mathrm{t}, 2 \mathrm{H}, J 7.5 \mathrm{~Hz}), 7.12(\mathrm{~d}, 2 \mathrm{H}, J 16.4 \mathrm{~Hz}, 2 \times \mathrm{H}-\alpha$ ), 7.24 (t, 2H, partially merged with $\mathrm{CHCl}_{3}$ peak), 7.38 (dd, $2 \mathrm{H}, J \quad 7.5$ and $1.5 \mathrm{~Hz}$ ), 7.43 (br. t, $2 \mathrm{H}, J 8.1 \mathrm{~Hz}), 7.57$ (d, $2 \mathrm{H}, J 16.4 \mathrm{~Hz}, 2 \times \mathrm{H}-\beta), 7.62$ (br. d, $2 \mathrm{H}, J 7.5 \mathrm{~Hz}) ;{ }^{13} \mathrm{C}$ NMR $(75 \mathrm{MHz}$, $\left.\mathrm{CDCl}_{3}\right) \delta_{\mathrm{C}}: 25.18,66.16,67.00,111.84,111.94,120.53,121.47,123.78,128.29,128.71,129.31$, 
129.50, 131.58, 131.72, 139.98, 156.64, 157.00, 196.37 (CO); TOFMSES $^{+}: \mathrm{m} / z \quad 583.08$ $(\mathrm{M}+\mathrm{Na})^{+}$. Anal. Calcd for $\mathrm{C}_{36} \mathrm{H}_{32} \mathrm{O}_{6}(560.64)$ : C, 77.12; H, 5.75\%. Found C, 77.18; H, 5.93\% . $(2 E, 25 E)-11,17,33,36$-Tetraoxapentacyclo[35.4.0.0 $\left.{ }^{5,10} \cdot 0^{18,23} \cdot 0^{27,32}\right]$ untetraconta-1(41),2,5,7,9,$\mathbf{1 8 , 2 0 , 2 2 , 2 5 , 2 7 , 2 9 , 3 1 , 3 7 , 3 9 - t e t r a d e c a e n e - 4 , 2 4 - d i o n e ~ ( 3 d ) . ~ E l u e n t : ~ p e t r o l e u m ~ e t h e r - E t O A c ~ ( 6 : 4 , ~}$ v/v), light yellow cubes, mp 218-220 ${ }^{\circ} \mathrm{C}$; IR (KBr): 1634 (CO), 1596, 1488, 1473, $1456 \mathrm{~cm}^{-1} ;{ }^{1} \mathrm{H}$ NMR $\left(300 \mathrm{MHz}, \mathrm{CDCl}_{3}\right) \delta_{\mathrm{H}}: 1.36-1.46\left(\mathrm{~m}, 6 \mathrm{H},-\mathrm{CH}_{2}-\underline{\mathrm{C}}_{2}-\underline{\mathrm{C}}_{2}-\underline{\mathrm{C}}_{2}-\mathrm{CH}_{2}-\right), 3.47$ (t, 4H, J 5.2 $\mathrm{Hz},-\underline{\mathrm{C}}_{2}-\mathrm{CH}_{2}-\mathrm{CH}_{2}-\mathrm{CH}_{2}-\underline{\mathrm{C}}_{2}-$ ), 4.26 (br. s, 4H, $\left.-\mathrm{CH}_{2}-\mathrm{CH}_{2}-\right), 6.57$ (d, 2H, J 8.2 Hz), 6.81 (d, 2H, $J \quad 8.2 \mathrm{~Hz}), 7.00(\mathrm{t}, 2 \mathrm{H}, J$ 7.4 Hz), $7.03(\mathrm{t}, 2 \mathrm{H}, J$ 7.5 Hz), 7.29-7.36 (m, 4H), 7.37 (d, 2H, J 16.2 $\mathrm{Hz}, 2 \times \mathrm{H}-\alpha)$ and 7.46 (br. d, 2H, J 7.5 Hz), $7.56(\mathrm{~d}, 2 \mathrm{H}, J 7.6 \mathrm{~Hz}), 7.66(\mathrm{~d}, 2 \mathrm{H}, J 16.2 \mathrm{~Hz}, 2 \times$ $\mathrm{H}-\beta) ;{ }^{13} \mathrm{C}$ NMR $\left(75 \mathrm{MHz}, \mathrm{CDCl}_{3}\right) \delta_{\mathrm{C}}: 23.38,29.05,67.66,68.08,112.01,112.81,120.47$, $121.54,124.28,129.01,129.65,130.02,130.30,131.42,132.05,139.39,157.11,157.66,195.69$ (CO); TOFMSES ${ }^{+}: m / z 597.00(\mathrm{M}+\mathrm{Na})^{+}$; Anal. Calcd for $\mathrm{C}_{37} \mathrm{H}_{34} \mathrm{O}_{6}(574.24)$ : C 77.33, H, 5.96\%. Found C, 77.09; H, 6.14\%.

$(2 E, 22 E)-11,14,30,34-T e t r a o x a p e n t a c y c l o\left[33.4 .0 .0^{5,10} \cdot 0^{15,20} \cdot 0^{24,29}\right]$ nonatriaconta-1(39),2,5,7,$\mathbf{9 , 1 5 , 1 7 , 1 9 , 2 2 , 2 4 , 2 6 , 2 8 , 3 5 , 3 7 - t e t r a d e c a e n e - 4 , 2 1 - d i o n e ~ ( 3 e ) . ~ E l u e n t : ~ p e t r o l e u m ~ e t h e r - E t O A c ~}$ (6:4, v/v), light yellow cubes, mp 186-188 ${ }^{\circ} \mathrm{C}$; IR (KBr): 1625 (CO), 1598, 1486, 1470, $1310 \mathrm{~cm}^{-}$ 1; ${ }^{1} \mathrm{H}$ NMR (300 MHz, $\mathrm{CDCl}_{3}$ ) $\delta_{\mathrm{H}}: 2.19$ (quintet, $\left.2 \mathrm{H}, J 6.0 \mathrm{~Hz},-\mathrm{CH}_{2}-\mathrm{CH}_{2}-\mathrm{CH}_{2}-\right), 4.09$ (t, 4H, $\left.6.0 \mathrm{~Hz},-\mathrm{CH}_{2}-\mathrm{CH}_{2}-\mathrm{CH}_{2}-\right), 4.27\left(\mathrm{~s}, 4 \mathrm{H},-\mathrm{CH}_{2}-\mathrm{CH}_{2}-\right), 6.78(\mathrm{~d}, 2 \mathrm{H}, J \quad 8.1 \mathrm{~Hz}), 6.80(\mathrm{~d}, 2 \mathrm{H}, J$ $8.1 \mathrm{~Hz}), 6.91(\mathrm{t}, 2 \mathrm{H}, J 7.4 \mathrm{~Hz}), 6.99$ (t, 2H, J 7.3 Hz), 7.26 (br. t, 4H, J 7.2 Hz), 7.43-7.47 (m, 4H), $7.48(\mathrm{~d}, 2 \mathrm{H}, J 16.5 \mathrm{~Hz}, 2 \times \mathrm{H}-\alpha), 7.64(\mathrm{~d}, 2 \mathrm{H}, J 16.2 \mathrm{~Hz}, 2 \times \mathrm{H}-\beta) ;{ }^{13} \mathrm{C} \mathrm{NMR}(75 \mathrm{MHz}$, $\left.\mathrm{CDCl}_{3}\right) \delta_{\mathrm{C}}: 28.41,63.94,68.17,112.11,113.67,121.03,121.55,124.05,128.90,129.96,130.25$, 131.05, 131.21, 132.28, 140.15, 156.71, 157.43, 194.73 (CO); FABMS: $m / z 547(\mathrm{M}+\mathrm{H})^{+}$; Anal. Calcd for $\mathrm{C}_{35} \mathrm{H}_{30} \mathrm{O}_{6}(546.61)$ : C, 76.91; H, 5.53\%. Found C, 77.02; H, 5.66\%.

$(2 E, 23 E)-11,15,31,35-T e t r a o x a p e n t a c y c l o\left[34.4 .0 .0^{5,10} \cdot 0^{16,21} .0^{25,30}\right]$ tetraconta-1(40),2,5,7,9,16,18,20,23,25,27,29,36,38-tetradecaene-4,22-dione (3f). Eluent: petroleum ether-EtOAc (6:4, v/v), light yellow cubes, mp 173-175 ${ }^{\circ} \mathrm{C}$; IR (KBr): 1634 (CO), 1594, 1482, 1471, $1310 \mathrm{~cm}^{-1} ;{ }^{1} \mathrm{H}$ NMR $\left(300 \mathrm{MHz}, \mathrm{CDCl}_{3}\right) \delta_{\mathrm{H}}: 2.08-2.17\left(\mathrm{~m}, 4 \mathrm{H}, 2 \times-\mathrm{CH}_{2}-\mathrm{CH}_{2}-\mathrm{CH}_{2}-\right), 3.96(\mathrm{t}, 4 \mathrm{H}, J 5.9 \mathrm{~Hz}$, $\left.\underline{\mathrm{CH}}_{2}-\mathrm{CH}_{2}-\underline{\mathrm{C}}_{2}-\right), 4.02$ (t, 4H, J $5.9 \mathrm{~Hz},-\underline{\mathrm{C}}_{2}-\mathrm{CH}_{2}-\underline{\mathrm{CH}}_{2}-$ ), 6.38 (d, 2H, J $8.1 \mathrm{~Hz}$ ), 6.76 (br. d, $2 \mathrm{H}, J 8.2 \mathrm{~Hz}), 6.92(\mathrm{t}, 2 \mathrm{H}, J 7.2 \mathrm{~Hz}), 7.00(\mathrm{t}, 2 \mathrm{H}, J 7.5 \mathrm{~Hz}), 7.10(\mathrm{dt}, 2 \mathrm{H}, J 6.9$ and $1.5 \mathrm{~Hz})$, $7.28(\mathrm{dt}, 2 \mathrm{H}, J$ 7.2 and $1.4 \mathrm{~Hz}), 7.35(\mathrm{~d}, 2 \mathrm{H}, J 16.2 \mathrm{~Hz}, 2 \times \mathrm{H}-\alpha), 7.42(\mathrm{dd}, 2 \mathrm{H}, J \quad 7.8$ and 1.6 $\mathrm{Hz}$ ), 7.56 (br. d, $2 \mathrm{H}, J 7.8 \mathrm{~Hz}), 7.67$ (d, $2 \mathrm{H}, J 16.2 \mathrm{~Hz}, 2 \times \mathrm{H}-\beta) ;{ }^{13} \mathrm{C} \mathrm{NMR}\left(75 \mathrm{MHz}, \mathrm{CDCl}_{3}\right) \delta_{\mathrm{C}}$ : 28.93, 29.36, 63.71, 64.89, 111.89, 112.36, 120.60, 121.20, 123.96, 128.76, 129.67, 129.72, 130.57, 131.52, 132.23, 140.40, 156.42, 157.47, 195.69 (CO); TOFMSES ${ }^{+}: \mathrm{m} / z .583$ [M+Na] Anal. Calcd for $\mathrm{C}_{36} \mathrm{H}_{32} \mathrm{O}_{6}(560.22)$ : C, 77.12; H, 5.75\%. Found C, 76.85, H, 5.71\%.

$(2 E, 24 E)-11,16,32,36$-Tetraoxapentacyclo[35.4.0.0 $\left.0^{5,10} \cdot 0^{17,22} \cdot 0^{26,31}\right]$ untetraconta-1(41),2,5,7,9,17,19,21,24,26,28,30,37,39-tetradecaene-4,23-dione (3g). Eluent: petroleum ether-EtOAc (1:1, v/v), light yellow cubes, mp 156-158 ${ }^{\circ} \mathrm{C}$; IR (KBr): 1630 (CO), 1484, 1448, 1387, $1308 \mathrm{~cm}^{-1} ;{ }^{1} \mathrm{H}$ NMR (300 MHz, $\mathrm{CDCl}_{3}$ ) $\delta_{\mathrm{H}}: 1.67$ (br. s, $4 \mathrm{H}, \mathrm{w}_{1 / 2} \approx 13.0 \mathrm{~Hz},-\mathrm{CH}_{2}-\underline{\mathrm{C}}_{2}-\mathrm{C}_{2}-\mathrm{CH}_{2}$ ), 2.21 (quintet, $\left.2 \mathrm{H}, J 5.9 \mathrm{~Hz},-\mathrm{CH}_{2}-\underline{\mathrm{C}}_{2}-\mathrm{CH}_{2}-\right), 3.63$ (br. s, $\left.4 \mathrm{H}, \mathrm{w}_{1 / 2} \approx 13.0 \mathrm{~Hz},-\mathrm{C}_{2}-\mathrm{CH}_{2}-\mathrm{CH}_{2}-\mathrm{CH}_{2}-\right), 4.15$ (t, $\left.4 \mathrm{H}, J 5.9 \mathrm{~Hz},-\underline{\mathrm{CH}}_{2}-\mathrm{CH}_{2}-\underline{\mathrm{CH}}_{2}-\right), 6.61(\mathrm{~d}, 2 \mathrm{H}, J 8.2 \mathrm{~Hz}), 6.84(\mathrm{~d}, 2 \mathrm{H}, J$ 8.2 Hz), 6.93-7.03 (m, 
4H), 7.24-7.36 (m, 4H), $7.36(\mathrm{~d}, 2 \mathrm{H}, J 16.2 \mathrm{~Hz}, 2 \times \mathrm{H}-\alpha), 7.49$ (dd, 2H, J 7.2 and 1.5 Hz), 7.52 $(\mathrm{dd}, 2 \mathrm{H}, J \quad 7.5$ and $1.6 \mathrm{~Hz}), 7.62(\mathrm{~d}, 2 \mathrm{H}, J 16.2 \mathrm{~Hz}, 2 \times \mathrm{H}-\beta) ;{ }^{13} \mathrm{C} \mathrm{NMR}\left(75 \mathrm{MHz}, \mathrm{CDCl}_{3}\right) \delta_{\mathrm{C}}$ : 26.13, 28.76, 63.85, 68.17, 112.19, 112.38, 120.80, 120.94, 124.16, 129.38, 129.65, 130.02, 130.46, 131.29, 132.34, 139.12, 157.12, 157.46, 194.82 (CO); TOFMSES ${ }^{+}: \mathrm{m} / z \quad 596.97$ $(\mathrm{M}+\mathrm{Na})^{+}$; Anal. Calcd for $\mathrm{C}_{37} \mathrm{H}_{34} \mathrm{O}_{6}$ (574.24): C, 77.33; H, 5.96\%. Found C, 77.09; H, 6.15\%. $(2 E, 25 E)-11,17,33,37-T e t r a o x a p e n t a c y c l o\left[36.4 .0 .0^{5,10} \cdot 0^{18,23} \cdot 0^{27,32}\right]$ dotetraconta-1(42),2,5,7,9,18,20,22,25,27,29,31,38,40-tetradecaene-4,24-dione (3h). Eluent: petroleum ether-EtOAc (1:1, v/v), light yellow cubes, mp 182-184 ${ }^{\circ} \mathrm{C}$; IR (KBr): 1630 (CO), 1586, 1530, 1485, $1407 \mathrm{~cm}^{-1} ;{ }^{1} \mathrm{H}$ NMR (300 MHz, $\mathrm{CDCl}_{3}$ ) $\delta_{\mathrm{H}}: 1.14$ (quintet, $2 \mathrm{H}, J \quad 6.0 \mathrm{~Hz},-\mathrm{CH}_{2}-\mathrm{CH}_{2}-\mathrm{CH}_{2}-\mathrm{CH}_{2}-\mathrm{CH}_{2}-$ ), 1.30 (br. $\mathrm{s}, 4 \mathrm{H}, \mathrm{w}_{1 / 2} \approx 24.0 \mathrm{~Hz},-\mathrm{CH}_{2}-\underline{\mathrm{CH}}_{2}-\mathrm{CH}_{2}-\mathrm{C}_{2}-\mathrm{CH}_{2}$ ), 2.13 (quintet, $2 \mathrm{H}, \mathrm{J} 5.1 \mathrm{~Hz},-\mathrm{CH}_{2}-\mathrm{CH}_{2}-\mathrm{CH}_{2}-$ ), 3.29 (br. t, $4 \mathrm{H}, J 5.0 \mathrm{~Hz},-\underline{\mathrm{CH}}_{2}-\mathrm{CH}_{2}-\mathrm{CH}_{2}-\mathrm{CH}_{2}-\underline{\mathrm{C}}_{2}-$ ), 4.06 (br. t, $4 \mathrm{H}, \mathrm{J} 5.4 \mathrm{~Hz},-\underline{\mathrm{C}}_{2}-\mathrm{CH}_{2}-\mathrm{C}_{2}-$ ), $6.67(\mathrm{~d}, 2 \mathrm{H}, J 8.4 \mathrm{~Hz}), 6.76(\mathrm{~d}, 2 \mathrm{H}, J 8.2 \mathrm{~Hz}), 6.97(\mathrm{t}, 2 \mathrm{H}, J 7.5 \mathrm{~Hz}), 7.04(\mathrm{t}, 2 \mathrm{H}, J 7.3 \mathrm{~Hz})$, 7.27 (br. t, $2 \mathrm{H}, J 8.1 \mathrm{~Hz}$ ), 7.32 (d, 2H, $J 16.1 \mathrm{~Hz}, 2 \times \mathrm{H}-\alpha$ ), 7.46 (dt, 2H, $J$ 7.6 and 1.4 Hz), 7.53 (br. d, 2H, $J$ 8.4 Hz), $7.56(\mathrm{dd}, 2 \mathrm{H}, J 7.6$ and $1.5 \mathrm{~Hz}), 7.62(\mathrm{~d}, 2 \mathrm{H}, J 16.1 \mathrm{~Hz}, 2 \times \mathrm{H}-\beta) ;{ }^{13} \mathrm{C}$ NMR $\left(75 \mathrm{MHz}, \mathrm{CDCl}_{3}\right) \delta_{\mathrm{C}}: 23.23,29.00,29.37,63.50,67.64,112.06,112.14,120.54,120.92$, 124.08, 129.45, 129.58, 130.15, 130.21, 131.31, 132.21, 138.95, 157.12, 157.57, 195.18 (CO); TOFMSES ${ }^{+}: m / z 611.24(\mathrm{M}+\mathrm{Na})^{+}$; Anal. Calcd for $\mathrm{C}_{38} \mathrm{H}_{36} \mathrm{O}_{6}(588.69)$ : C, 77.53; H, 6.16\% . Found C, 77.36; H, 6.37\%.

\section{$(2 E, 22 E)-11,14,30,35-T e t r a o x a p e n t a c y c l o\left[34.4 .0 .0^{5,10} \cdot 0^{15,20} \cdot 0^{24,29}\right]$ tetraconta-1(40),2,5,7,9,-}

15,17,19,22,24, 26,28,36,38-tetradecaene-4,21-dione (3i). Eluent: petroleum ether-EtOAc (1:1, v/v), light yellow cubes, mp 148-150 ${ }^{\circ} \mathrm{C}$; IR (KBr): 1625 (CO), 1486, 1448, 1387, $1310 \mathrm{~cm}^{-1} ;{ }^{1} \mathrm{H}$ NMR $\left(300 \mathrm{MHz}, \mathrm{CDCl}_{3}\right) \delta_{\mathrm{H}}: 1.83$ (br. s, $4 \mathrm{H}, \mathrm{w}_{1 / 2} \approx 12.5 \mathrm{~Hz},-\mathrm{CH}_{2}-\underline{\mathrm{C}}_{2}-\mathrm{C}_{2}-\mathrm{CH}_{2}$ ), 3.94 (br. s, $\left.4 \mathrm{H}, \mathrm{w}_{1 / 2} \approx 12.5 \mathrm{~Hz},-\mathrm{CH}_{2}-\mathrm{CH}_{2}-\mathrm{CH}_{2}-\underline{\mathrm{CH}}_{2}-\right), 4.27$ (s, 4H, $\left.-\mathrm{CH}_{2}-\mathrm{CH}_{2}-\right), 6.74$ (d, 2H, J 8.1 Hz), 6.89 $(\mathrm{d}, 2 \mathrm{H}, J 8.1 \mathrm{~Hz}), 6.98(\mathrm{t}, 2 \mathrm{H}, J 8.4 \mathrm{~Hz}), 7.03(\mathrm{t}, 2 \mathrm{H}, J 7.5 \mathrm{~Hz}), 7.19$ (dt, 2H, $J 7.5 \mathrm{and} 1.8 \mathrm{~Hz})$, $7.38(\mathrm{dt}, 2 \mathrm{H}, J 7.8$ and $1.5 \mathrm{~Hz})$ and $7.41(\mathrm{~d}, 2 \mathrm{H}, J 16.5 \mathrm{~Hz}, 2 \times \mathrm{H}-\alpha), 7.45(\mathrm{dd}, 2 \mathrm{H}, J 7.8$ and 1.8 $\mathrm{Hz}$ ), $7.61(\mathrm{dd}, 2 \mathrm{H}, J 7.8$ and $1.8 \mathrm{~Hz}), 7.70$ (d, $2 \mathrm{H}, J 16.5 \mathrm{~Hz}, 2 \times \mathrm{H}-\beta) ;{ }^{13} \mathrm{C} \mathrm{NMR}(75 \mathrm{MHz}$, $\left.\mathrm{CDCl}_{3}\right) \delta_{\mathrm{C}}: 26.11,67.04,67.94,111.87,113.30,121.01,121.37,123.65,128.64,129.78,130.28$, 130.62, 131.57, 131.91, 140.69, 156.19, 157.92, $195.43(\mathrm{CO})$; FABMS: $m / z .561(\mathrm{M}+\mathrm{H})^{+}, 583$ $(\mathrm{M}+\mathrm{Na})^{+}$; Anal. Calcd for $\mathrm{C}_{36} \mathrm{H}_{32} \mathrm{O}_{6}(560.64)$ : C, 77.12; H, 5.75\%. Found C, 76.88; H, 6.02\%.

$(2 E, 23 E)-11,15,31,36$-Tetraoxapentacyclo[35.4.0.0 $\left.{ }^{5,10} \cdot 0^{16,21} \cdot 0^{25,30}\right]$ untetraconta-1(41),2,5,7,9,16,18,20, 23, 25,27,29,37,39-tetradecaene-4,22-dione (3j). Eluent: petroleum ether-EtOAc (1:1, v/v), light yellow cubes, mp 198-200 ${ }^{\circ} \mathrm{C}$; IR (KBr): 1633 (CO), 1596, 1488, 1473, $1390 \mathrm{~cm}^{-1} ;{ }^{1} \mathrm{H}$ NMR (300 MHz, $\mathrm{CDCl}_{3}$ ) $\delta_{\mathrm{H}}: 1.67$ (br. s, $4 \mathrm{H}, \mathrm{w}_{1 / 2} \approx 12.0 \mathrm{~Hz},-\mathrm{CH}_{2}-\underline{\mathrm{C}}_{2}-\mathrm{C}_{2}-\mathrm{CH}_{2}$ ), 2.06 (quintet, $\left.2 \mathrm{H}, J 5.4 \mathrm{~Hz},-\mathrm{CH}_{2}-\underline{\mathrm{C}}_{2}-\mathrm{CH}_{2}-\right), 3.81$ (br. s, $4 \mathrm{H}, \mathrm{w}_{1 / 2} \approx 12.0 \mathrm{~Hz},-\mathrm{C}_{2}-\mathrm{CH}_{2}-\mathrm{CH}_{2}-\mathrm{C}_{2}-$ ), 4.00 (t, $\left.4 \mathrm{H}, J 5.4 \mathrm{~Hz},-\underline{\mathrm{CH}}_{2}-\mathrm{CH}_{2}-\underline{\mathrm{CH}}_{2}-\right), 6.48(\mathrm{~d}, 2 \mathrm{H}, J 8.1 \mathrm{~Hz}), 6.86(\mathrm{~d}, 2 \mathrm{H}, J 8.4 \mathrm{~Hz}), 6.88(\mathrm{t}, 2 \mathrm{H}, J$ $7.5 \mathrm{~Hz}), 6.99(\mathrm{~d}, 2 \mathrm{H}, J 1.7 \mathrm{~Hz}), 7.04(\mathrm{t}, 2 \mathrm{H}, J$ 7.5 Hz), $7.28(\mathrm{~d}, 2 \mathrm{H}, J 16.5 \mathrm{~Hz}, 2 \times \mathrm{H}-\alpha), 7.30$ (dd, 2H, $J 7.1$ and $1.8 \mathrm{~Hz}$ ), 7.39 (dt, 2H, $J 8.6$ and $1.5 \mathrm{~Hz}), 7.61(\mathrm{~d}, 2 \mathrm{H}, J 1.5 \mathrm{~Hz}), 7.66(\mathrm{~d}, 2 \mathrm{H}$, $J 16.4 \mathrm{~Hz}, 2 \times \mathrm{H}-\beta) ;{ }^{13} \mathrm{C} \mathrm{NMR}\left(75 \mathrm{MHz}, \mathrm{CDCl}_{3}\right) \delta_{\mathrm{C}}: 26.00,29.34,64.49,67.78,111.92,112.41$, $120.26,121.07,123.72$, 128.69, 128.92, 129.92, 130.04, 131.38, 131.73, 140.93, 156.27, 157.88, 
196.66 (CO); FABMS: $m / z, 596.97(\mathrm{M}+\mathrm{Na})^{+}$; Anal. Calcd for $\mathrm{C}_{37} \mathrm{H}_{34} \mathrm{O}_{6}$ (574.24): C, 77.33; $\mathrm{H}$, $5.96 \%$. Found C, 77.11; H, 6.18\%.

$(2 E, 24 E)-11,16,32,37-T e t r a o x a p e n t a c y c l o\left[36.4 .0 .0^{5,10} \cdot 0^{17,22} \cdot 0^{26,31}\right]$ dotetraconta-1(42),2,5,7,9,$\mathbf{1 7 , 1 9 , 2 1 , 2 4 , ~ 2 6 , 2 8 , 3 0 , 3 8 , 4 0 - t e t r a d e c a e n e - 4 , 2 3 - d i o n e ~ ( 3 k ) . ~ E l u e n t : ~ p e t r o l e u m ~ e t h e r - E t O A c ~ ( 1 : 1 , ~}$ v/v), light yellow cubes, mp 162-164 ${ }^{\circ} \mathrm{C}$; IR (KBr): 1631 (CO), 1489, 1448, 1387, $1309 \mathrm{~cm}^{-1} ;{ }^{1} \mathrm{H}$ NMR (300 MHz, $\left.\mathrm{CDCl}_{3}\right) \delta_{\mathrm{H}}: 1.74$ (br. s, 8H, w $\left.1 / 2 \approx 12.5 \mathrm{~Hz}, 2 \times-\mathrm{CH}_{2}-\mathrm{CH}_{2}-\mathrm{CH}_{2}-\mathrm{CH}_{2}-\right), 3.78$ (br. s, $4 \mathrm{H}, \mathrm{w}_{1 / 2} \approx 12.0 \mathrm{~Hz},-\underline{\mathrm{C}}_{2}-\mathrm{CH}_{2}-\mathrm{CH}_{2}-\underline{\mathrm{CH}}_{2}-$ ), 3.97 (br. s, $4 \mathrm{H}, \mathrm{w}_{1 / 2} \approx 12.0 \mathrm{~Hz},-\underline{\mathrm{C}}_{2}-\mathrm{CH}_{2}-\mathrm{CH}_{2}-\mathrm{C}_{2}-$ ), $6.60(\mathrm{~d}, 2 \mathrm{H}, J 8.4 \mathrm{~Hz}), 6.91(\mathrm{~d}, 2 \mathrm{H}, J 8.4 \mathrm{~Hz}), 6.96(\mathrm{t}, 2 \mathrm{H}, J 7.5 \mathrm{~Hz}), 6.98(\mathrm{t}, 2 \mathrm{H}, J 7.5 \mathrm{~Hz})$, $7.18(\mathrm{~d}, 2 \mathrm{H}, J 16.2 \mathrm{~Hz}, 2 \times \mathrm{H}-\alpha), 7.25\left(\mathrm{dt}, 2 \mathrm{H}\right.$, partially merged with $\mathrm{CHCl}_{3}$ peak), 7.37 (dt, $2 \mathrm{H}$, $J 7.5$ and $1.5 \mathrm{~Hz}$ ), 7.38 (dd, 2H, $J 7.5$ and $1.5 \mathrm{~Hz}$ ), 7.59 (dd, 2H, $J 7.8$ and $1.2 \mathrm{~Hz}$ ), 7.66 (d, 2H, $J 16.2 \mathrm{~Hz}, 2 \times \mathrm{H}-\beta) ;{ }^{13} \mathrm{C} \mathrm{NMR}\left(75 \mathrm{MHz}, \mathrm{CDCl}_{3}\right) \delta_{\mathrm{C}}: 25.12,25.98,66.98,67.70,111.94,112.33$, 120.52, 120.99, 123.83, 128.39, 129.16, 129.43, 129.92, 131.54, 131.73, 140.43, 156.58, 157.58, 196.02 (CO); TOFMSES ${ }^{+}: m / z 611.24(\mathrm{M}+\mathrm{Na})^{+}$; Anal. Calcd for $\mathrm{C}_{38} \mathrm{H}_{36} \mathrm{O}_{6}$ (588.25): C, 77.53; H, 6.16\%. Found C, 77.40; H, 6.06\%.

$(2 E, 25 E)-11,17,33,38$-Tetraoxapentacyclo[37.4.0.0 $\left.0^{5,10} \cdot 0^{18,23} \cdot 0^{27,32}\right]$ tritetraconta-1(43),2,5,7,9,$\mathbf{1 8 , 2 0 , 2 2 , 2 5 , 2 7 , 2 9 , 3 1 , 3 9 , 4 1 - t e t r a d e c a e n e - 4 , 2 4 - d i o n e ~ ( 3 l ) . ~ E l u e n t : ~ p e t r o l e u m ~ e t h e r - E t O A c ~ ( 1 : 1 , ~}$ $\mathrm{v} / \mathrm{v})$, light yellow cubes, mp 173-175 ${ }^{\circ} \mathrm{C}$; IR (KBr): 1630 (CO), 1586, 1483, 1446, $1389 \mathrm{~cm}^{-1} ;{ }^{1} \mathrm{H}$ NMR $\left(300 \mathrm{MHz}, \mathrm{CDCl}_{3}\right) \delta_{\mathrm{H}}: 1.26-1.33\left(\mathrm{~m}, 2 \mathrm{H},-\mathrm{CH}_{2}-\mathrm{CH}_{2}-\underline{\mathrm{C}}_{2}-\mathrm{CH}_{2}-\mathrm{CH}_{2}-\right), 1.53$ (quintet, 4H, $J$ $6.9 \mathrm{~Hz},-\mathrm{CH}_{2}-\underline{\mathrm{CH}}_{2}-\mathrm{CH}_{2}-\underline{\mathrm{CH}}_{2}-\mathrm{CH}_{2}-$ ), 1.86 (br. s, $4 \mathrm{H}, \mathrm{w}_{1 / 2} \approx 12.5 \mathrm{~Hz},-\mathrm{CH}_{2}-\mathrm{CH}_{2}-\mathrm{CH}_{2}-\mathrm{CH}_{2}-$ ), 3.69 (t, $4 \mathrm{H}, J$ 5.7Hz, $-\underline{\mathrm{CH}}_{2}-\mathrm{CH}_{2}-\mathrm{CH}_{2}-\mathrm{CH}_{2}-\underline{\mathrm{C}}_{2}-$ ), 3.99 (br. s, $4 \mathrm{H}, \mathrm{w}_{1 / 2} \approx 12.5 \mathrm{~Hz},-\underline{\mathrm{C}}_{2}-\mathrm{CH}_{2}-\mathrm{CH}_{2}-\mathrm{C}_{2}-$ ), $6.71(\mathrm{~d}, 2 \mathrm{H}, J 8.3 \mathrm{~Hz}), 6.85(\mathrm{~d}, 2 \mathrm{H}, J 8.2 \mathrm{~Hz}), 6.92(\mathrm{t}, 2 \mathrm{H}, J 7.5 \mathrm{~Hz}), 7.00(\mathrm{t}, 2 \mathrm{H}, J 7.4 \mathrm{~Hz})$, $7.24(\mathrm{t}, 2 \mathrm{H}, J \approx 8.4 \mathrm{~Hz}), 7.30(\mathrm{~d}, 2 \mathrm{H}, J 16.3 \mathrm{~Hz}, 2 \times \mathrm{H}-\alpha), 7.35(\mathrm{t}, 2 \mathrm{H}, J 7.5 \mathrm{~Hz}), 7.48(\mathrm{~d}, 2 \mathrm{H}, J$ $7.4 \mathrm{~Hz}), 7.53(\mathrm{~d}, 2 \mathrm{H}, J 7.6 \mathrm{~Hz}), 7.73(\mathrm{~d}, 2 \mathrm{H}, J 16.1 \mathrm{~Hz}, 2 \times \mathrm{H}-\beta) ;{ }^{13} \mathrm{C}$ NMR $\left(75 \mathrm{MHz}, \mathrm{CDCl}_{3}\right)$ $\delta_{\mathrm{C}}: 22.70,26.11,28.56,68.05,68.40,112.13,112.53,120.51,120.97,124.19,128.62,129.57$, 129.77, 130.01, 131.53, 132.04, 139.47, 157.03, 157.89, 195.30 (CO); GCMS: m/z 602(M+). Anal. Calcd for $\mathrm{C}_{39} \mathrm{H}_{38} \mathrm{O}_{6}$ (602.27): C, 77.72; H, 6.35\%. Found C, 77.48; H, 6.40\%.

$(2 E, 22 E)-11,14,30,36-T e t r a o x a p e n t a c y c l o\left[35.4 .0 .0^{5,10} \cdot 0^{15,20} \cdot 0^{24,29}\right]$ untetraconta-1(41),2,5,7,9,$\mathbf{1 5 , 1 7 , 1 9 , 2 2 , 2 4 , 2 6 , 2 8 , 3 7 , 3 9 - t e t r a d e c a e n e - 4 , 2 1 - d i o n e ~ ( 3 m ) . ~ E l u e n t : ~ p e t r o l e u m ~ e t h e r - E t O A c ~ ( 1 : 1 , ~}$ v/v), light yellow cubes, mp 212-214 ${ }^{\circ} \mathrm{C}$; IR (KBr): 1634 (CO), 1597, 1488, 1473, $1390 \mathrm{~cm}^{-1} ;{ }^{1} \mathrm{H}$ NMR $\left(300 \mathrm{MHz}, \mathrm{CDCl}_{3}\right.$ ) $\delta_{\mathrm{H}}: 1.54$ (quintet, $2 \mathrm{H}, \mathrm{J} 6.6 \mathrm{~Hz},-\mathrm{CH}_{2}-\mathrm{CH}_{2}-\mathrm{CH}_{2}-\mathrm{CH}_{2}-\mathrm{CH}_{2}-$ ), 1.69-1.78 (m, $\left.4 \mathrm{H},-\mathrm{CH}_{2}-\underline{\mathrm{CH}}_{2}-\mathrm{CH}_{2}-\underline{\mathrm{C}}_{2}-\mathrm{CH}_{2}-\right), 3.88\left(\mathrm{t}, 4 \mathrm{H}, J \quad 5.5 \mathrm{~Hz},-\mathrm{CH}_{2}-\mathrm{CH}_{2}-\mathrm{CH}_{2}-\mathrm{CH}_{2}-\mathrm{CH}_{2}-\right), 4.20$ (s, $\left.4 \mathrm{H},-\mathrm{CH}_{2}-\mathrm{CH}_{2}-\right), 6.56(\mathrm{~d}, 2 \mathrm{H}, J$ 8.4), $6.88(\mathrm{~d}, 2 \mathrm{H}, J 8.3 \mathrm{~Hz}), 6.97$ (t, 2H, J 7.8 Hz), 7.00 (t, 2H, $7.7 \mathrm{~Hz}$ ), 7.18 (dt, 2H, $J 7.2$ and $1.5 \mathrm{~Hz}$ ), 7.36 (dt, 2H, $J 7.2$ and $1.4 \mathrm{~Hz}$ ), 7.55 (br. d, 4H, $J 7.5$ $\mathrm{Hz}), 7.57$ (d, 2H, $J$ 16.2 Hz, $2 \times \mathrm{H}-\alpha), 7.67$ (d, $2 \mathrm{H}, J \quad 16.2 \mathrm{~Hz}, 2 \times \mathrm{H}-\beta) ;{ }^{13} \mathrm{C} \mathrm{NMR}(75 \mathrm{MHz}$, $\left.\mathrm{CDCl}_{3}\right) \delta_{\mathrm{C}}: 23.99,29.30,67.49,68.40,112.29,113.55,121.01,121.59,124.02,129.20,130.20$, 130.36, 131.07, 131.33, 132.50, 139.60, 156.80, 158.19, 194.45 (CO); EIMS: $\mathrm{m} / z .574\left(\mathrm{M}^{+}\right)$; Anal. Calcd for $\mathrm{C}_{37} \mathrm{H}_{34} \mathrm{O}_{6}$ (574.24): C, 77.33; H, 5.96\%. Found C, 77.23; H, 6.12\%.

$(2 E, 23 E)-11,15,31,37-T e t r a 0 x a p e n t a c y c l o\left[36.4 .0 .0^{5,10} \cdot 0^{16,21} \cdot 0^{25,30}\right]$ dotetraconta-1(42),2,5,7,9,$\mathbf{1 6 , 1 8 , 2 0 , 2 3 , 2 5 , ~ 2 7 , 2 9 , 3 8 , 4 0 - t e t r a d e c a e n e - 4 , 2 2 - d i o n e ~ ( 3 n ) . ~ E l u e n t : ~ p e t r o l e u m ~ e t h e r - E t O A c ~ ( 1 : 1 , ~}$ $\mathrm{v} / \mathrm{v})$, light yellow cubes, mp 148-150 ${ }^{\circ} \mathrm{C}$; IR (KBr): 1631 (CO), 1491, 1482, 1347, $1308 \mathrm{~cm}^{-1} ;{ }^{1} \mathrm{H}$ 
NMR (300 MHz, $\left.\mathrm{CDCl}_{3}\right) \delta_{\mathrm{H}}: 1.42-1.50\left(\mathrm{~m}, 2 \mathrm{H},-\mathrm{CH}_{2}-\mathrm{CH}_{2}-\mathrm{C}_{2}-\mathrm{CH}_{2}-\mathrm{CH}_{2}\right.$ ) , 1.57-1.65 (m, 4H, $\mathrm{CH}_{2}-\underline{\mathrm{C}}_{2}-\mathrm{CH}_{2}-\underline{\mathrm{C}}_{2}-\mathrm{CH}_{2}$ ), 1.96 (quintet, $2 \mathrm{H}, J 5.1 \mathrm{~Hz},-\mathrm{CH}_{2}-\mathrm{CH}_{2}-\mathrm{CH}_{2}-$ ), 3.74 (t, 4H, J $5.2 \mathrm{~Hz}$, $\left.-\underline{\mathrm{C}}_{2}-\mathrm{CH}_{2}-\mathrm{CH}_{2}-\mathrm{CH}_{2}-\underline{\mathrm{CH}}_{2}-\right), 3.84\left(\mathrm{t}, 4 \mathrm{H}, J \quad 5.2 \mathrm{~Hz},-\underline{\mathrm{C}}_{2}-\mathrm{CH}_{2}-\underline{\mathrm{C}}_{2}-\right.$ ), 6.22 (d, $\left.2 \mathrm{H}, \mathrm{J} \quad 8.2 \mathrm{~Hz}\right)$, $6.90(2 \mathrm{H}, \mathrm{d}, J 8.2 \mathrm{~Hz}), 6.98(\mathrm{t}, 2 \mathrm{H}, J 7.5 \mathrm{~Hz}), 7.06(\mathrm{t}, 2 \mathrm{H}, J 7.5 \mathrm{~Hz}), 7.19(\mathrm{dt}, 2 \mathrm{H}, J 7.2$ and 1.7 $\mathrm{Hz}), 7.42(\mathrm{dt}, 2 \mathrm{H}, J 7.2$ and $1.4 \mathrm{~Hz}), 7.49(\mathrm{dd}, 2 \mathrm{H}, J 7.5$ and $1.7 \mathrm{~Hz}), 7.50(\mathrm{~d}, 2 \mathrm{H}, J$ 16.2 Hz, 2 $\times \mathrm{H}-\alpha), 7.58(\mathrm{dd}, 2 \mathrm{H}, J 7.8$ and $1.2 \mathrm{~Hz}), 7.65(\mathrm{~d}, 2 \mathrm{H}, J \quad 16.1 \mathrm{~Hz}, 2 \times \mathrm{H}-\beta) ;{ }^{13} \mathrm{C} \mathrm{NMR}(75 \mathrm{MHz}$, $\left.\mathrm{CDCl}_{3}\right) \delta_{\mathrm{C}}: 24.37,29.62,29.65,64.19,68.44,111.64,112.36,120.50,120.96,123.88,129.77$, 129.94, 129.97, 131.21, 131.25, 132.28, 139.37, 156.75, 158.25, 195.36 (CO); TOFMSES ${ }^{+}: \mathrm{m} / z$ $611.03(\mathrm{M}+\mathrm{Na})^{+}$; Anal. Calcd for $\mathrm{C}_{38} \mathrm{H}_{36} \mathrm{O}_{6}(588.25)$ : C, 77.53; H, 6.16\%. Found C, 77.29; H , $6.31 \%$.

$2 E, 24 E)-11,16,32,38$-Tetraoxapentacyclo[37.4.0.0 $\left.0^{5,10} \cdot 0^{17,22} \cdot 0^{26,31}\right]$ tritetraconta-1(43),2,5,7,9,17,19,21,24,26, 28,30,39,41-tetradecaene-4,23-dione (3o). Eluent: petroleum ether-EtOAc (1:1, v/v), light yellow cubes, mp 205-207 ${ }^{\circ} \mathrm{C}$; IR (KBr): 1632 (CO), 1487, 1448, 1308, $1246 \mathrm{~cm}^{-1} ;{ }^{1} \mathrm{H}$ NMR $\left(300 \mathrm{MHz}, \mathrm{CDCl}_{3}\right) \delta_{\mathrm{H}}: 1.35-1.42\left(\mathrm{~m}, 2 \mathrm{H},-\mathrm{CH}_{2}-\mathrm{CH}_{2}-\mathrm{CH}_{2}-\mathrm{CH}_{2}-\mathrm{CH}_{2}-\right), 1.59$ (br. s, 4H, $\mathrm{w}_{1 / 2} \approx 12.0 \mathrm{~Hz},-\mathrm{CH}_{2}-\mathrm{CH}_{2}-\mathrm{C}_{2}-\mathrm{CH}_{2}-$ ), 1.67-1.74 (m, 4H, $-\mathrm{CH}_{2}-\underline{\mathrm{CH}}_{2}-\mathrm{CH}_{2}-\mathrm{C}_{2}-\mathrm{CH}_{2}$ ), 3.40 (br. s, $\left.4 \mathrm{H}, \mathrm{w}_{1 / 2} \approx 12.0 \mathrm{~Hz},-\underline{\mathrm{C}}_{2}-\mathrm{CH}_{2}-\mathrm{CH}_{2}-\underline{\mathrm{C}}_{2}-\right), 3.96\left(\mathrm{~s}, 4 \mathrm{H}, \mathrm{w}_{1 / 2} \approx 12.5 \mathrm{~Hz},-\underline{\mathrm{C}}_{2}-\mathrm{CH}_{2}-\mathrm{CH}_{2}-\mathrm{CH}_{2}-\mathrm{C}_{2}-\right.$ ), $6.44(\mathrm{~d}, 2 \mathrm{H}, J$ 8.1 Hz), 6.96-7.01 (m, 6H), $7.10(\mathrm{~d}, 2 \mathrm{H}, J 16.3 \mathrm{~Hz}, 2 \times \mathrm{H}-\alpha), 7.32(\mathrm{t}, 2 \mathrm{H}, J 7.2$ $\mathrm{Hz}), 7.38-7.42(\mathrm{~m}, 4 \mathrm{H}), 7.62(\mathrm{~d}, 2 \mathrm{H}, J \quad 6.9 \mathrm{~Hz}), 7.63(\mathrm{~d}, 2 \mathrm{H}, J 16.6 \mathrm{~Hz}, 2 \times \mathrm{H}-\beta) ;{ }^{13} \mathrm{C}$ NMR $(75$ $\left.\mathrm{MHz}, \mathrm{CDCl}_{3}\right) \delta_{\mathrm{C}}: 23.47,25.05,28.90,66.00,68.25,111.77,111.94,120.67,121.09,123.79$, 128.33, 128.53, 129.64, 131.66, 131.79, 133.65, 139.42, 156.49, 157.59, 196.26 (CO); GCMS: $m / z$ 602( $\left(\mathrm{M}^{+}\right)$; Anal. Calcd for $\mathrm{C}_{39} \mathrm{H}_{38} \mathrm{O}_{6}$ (602.27): C, 77.72; H, 6.35\%. Found $\mathrm{C}, 77.65 ; \mathrm{H}$, $6.60 \%$.

$(2 E, 25 E)-11,17,33,39-T e t r a o x a p e n t a c y c l o\left[38.4 .0 .0^{5,10} \cdot 0^{18,23} \cdot 0^{27,32}\right]$ tetratetraconta-1(44),2,5,7,$\mathbf{9 , 1 8 , 2 0 , 2 2 , 2 5 , 2 7 , 2 9 , 3 1 , 4 0 , 4 2 - t e t r a d e c a e n e - 4 , 2 4 - d i o n e ~ ( 3 p ) . ~ E l u e n t : ~ p e t r o l e u m ~ e t h e r - E t O A c ~}$ $(1: 1, \mathrm{v} / \mathrm{v})$, light yellow cubes, mp 212-214 ${ }^{\circ} \mathrm{C}$; IR (KBr): 1633 (CO), 1488, 1445, 1386, $1304 \mathrm{~cm}^{-}$ 1; ${ }^{1} \mathrm{H}$ NMR $\left(\mathrm{CDCl}_{3}\right) \delta_{\mathrm{H}}$ : 1.24-1.29 (m, 2H, $\left.-\mathrm{CH}_{2}-\mathrm{CH}_{2}-\mathrm{CH}_{2}-\mathrm{CH}_{2}-\mathrm{CH}_{2-}\right), 1.34-1.41$ (m, 4H, - $\mathrm{CH}_{2-}$ $\mathrm{CH}_{2}-\mathrm{CH}_{2}-\underline{\mathrm{CH}}_{2}-\mathrm{CH}_{2}-$ ), 1.52-1.59 (m, $2 \mathrm{H},-\mathrm{CH}_{2}-\mathrm{CH}_{2}-\mathrm{CH}_{2}-\mathrm{CH}_{2}-\mathrm{CH}_{2}-$ ), 1.71-1.78 (m, 4H, - $\mathrm{CH}_{2}-$ $\left.\mathrm{C}_{2}-\mathrm{CH}_{2}-\underline{\mathrm{CH}}_{2}-\mathrm{CH}_{2}-\right), 3.48\left(\mathrm{t}, 4 \mathrm{H}, J 5.2 \mathrm{~Hz},-\mathrm{CH}_{2}-\mathrm{CH}_{2}-\mathrm{CH}_{2}-\mathrm{CH}_{2}-\underline{C}_{2}-\right), 3.93$ (t, 4H, J $5.5 \mathrm{~Hz}$, $\left.\mathrm{C}_{2}-\mathrm{CH}_{2}-\mathrm{CH}_{2}-\mathrm{CH}_{2}-\underline{\mathrm{CH}}_{2}-\right), 6.64(\mathrm{~d}, 2 \mathrm{H}, J \quad 8.3 \mathrm{~Hz}), 6.84(\mathrm{~d}, 2 \mathrm{H}, J \quad 8.3 \mathrm{~Hz}), 6.94$ (2H, t, J 7.4 $\mathrm{Hz}$ ), 7.01 (t, 2H, J 7.4 Hz), 7.29 (d, 2H, J 7.3 Hz), 7.33 (d, 2H, J 16.2 Hz, $2 \times \mathrm{H}-\alpha), 7.39$ (dt, $2 \mathrm{H}, J 7.9$ and $1.2 \mathrm{~Hz}$ ), 7.50 (br. d, $2 \mathrm{H}, J$ 8.2 Hz), 7.52 (dt, 2H, $J 7.4 \mathrm{~Hz}$ and $1.5 \mathrm{~Hz}$ ), 7.70 (d, $2 \mathrm{H}, J 16.2 \mathrm{~Hz}, 2 \times \mathrm{H}-\beta) ;{ }^{13} \mathrm{C} \mathrm{NMR}\left(75 \mathrm{MHz}, \mathrm{CDCl}_{3}\right) \delta_{\mathrm{C}}: 18.46,19.06,24.16,24.45,63.27$, 63.86, 107.39, 107.70, 116.01, 116.24, 119.62, 124.65, 125.30, 125.48, 126.76, 127.60, 134.61, 152.66, 153.61, 190.51 (CO); FABMS: $m / z 617.4(\mathrm{M}+\mathrm{H})^{+}$; Anal. Calcd for $\mathrm{C}_{40} \mathrm{H}_{40} \mathrm{O}_{6}(616.74)$ : C, 77.90; H, 6.54\%. Found C, 77.77; H, 6.69\%.

General method for reaction of $2,2^{\prime}$-dihydroxychalcone (6) with $\alpha, \omega$-dibromoalkanes. To a solution of 2,2'-dihydroxychalcone $(6)(1 \mathrm{mmol})$ in dry acetone $(15 \mathrm{~mL}), \alpha, \omega$-dibromoalkane $(1$ mmol) and anhydrous $\mathrm{K}_{2} \mathrm{CO}_{3}(1.5 \mathrm{~g})$ were added and the mixture was refluxed for $10 \mathrm{hr}$. Usual work-up of the reaction mixture followed by purification of the resulting material by column 
chromatography and crystallization from $\mathrm{CHCl}_{3}$-petroleum ether afforded the cyclic compounds 7. The analytical and spectral data of 7a-c were as follows:

(2E)-11,15-Dioxatricyclo[14.4.0.0 $\left.{ }^{5,10}\right]$ eicosa-1(20),2,5,7,9,16,18-heptaene-4-one (7a). Eluent: petroleum ether-EtOAc (8:2, v/v), light yellow cubes, mp $96{ }^{\circ} \mathrm{C}$; IR (KBr): 1625 (CO), 1616, 1454, 1294, $1280 \mathrm{~cm}^{-1} ;{ }^{1} \mathrm{H} \mathrm{NMR}\left(300 \mathrm{MHz}, \mathrm{CDCl}_{3}\right) \delta_{\mathrm{H}}: 2.25$ (quintet, $2 \mathrm{H}, J 5.4 \mathrm{~Hz},-\mathrm{CH}_{2}-\mathrm{CH}_{2}-$

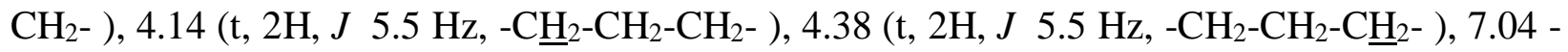
$7.18(\mathrm{~m}, 4 \mathrm{H}), 7.31(\mathrm{t}, 1 \mathrm{H}, J 8.6 \mathrm{~Hz}), 7.36(\mathrm{~d}, 1 \mathrm{H}, J 7.6 \mathrm{~Hz}), 7.49$ ( t, 1H, J $7.8 \mathrm{~Hz}), 7.52$ (d, 1H, $J 16.2 \mathrm{~Hz}, \mathrm{H}-\alpha$ ), 7.60 (d, 1H, J 16.2 Hz, H- $\beta$ ), 7.72 (br. d, 1H, J 7.5 Hz, proton ortho to CO); ${ }^{13} \mathrm{C}$ NMR $\left(75 \mathrm{MHz}, \mathrm{CDCl}_{3}\right) \delta_{\mathrm{C}}: 29.40,66.60,69.38,115.98,120.45,122.30,123.85,128.90$, 130.35, 130.56, 130.72, 131.54, 132.62, 133.23, 138.26, 158.12, 158.20, 193.01 (CO); GCMS: $m / z 280\left(\mathrm{M}^{+}\right)$; Anal. Calcd for $\mathrm{C}_{18} \mathrm{H}_{16} \mathrm{O}_{3}$ (280.31): C, 77.12; H, 5.75\%. Found C, 76.95; H, $5.91 \%$.

(2E)-11,16-Dioxatricyclo[15.4.0.0 $\left.{ }^{5,10}\right]$ uncosa-1(21),2,5,7,9,17,19-heptaene-4-one (7b). Eluent: petroleum ether-EtOAc (8:2, v/v), light yellow cubes, mp $123{ }^{\circ} \mathrm{C}$; IR (KBr): 1631 (CO), 1610, 1452, 1291, $1282 \mathrm{~cm}^{-1}$; ${ }^{1} \mathrm{H}$ NMR $\left(300 \mathrm{MHz}, \mathrm{CDCl}_{3}\right) \delta_{\mathrm{H}}: 2.18$ (br. s, 4H, w1/2 $12.0 \mathrm{~Hz},-\mathrm{CH}_{2^{-}}$ $\underline{\mathrm{C}}_{2}-\underline{\mathrm{C}}_{2}-\mathrm{CH}_{2}-$ ), 4.12 (br. s, $4 \mathrm{H}, \mathrm{w}_{1 / 2} \approx 13.5 \mathrm{~Hz},-\underline{\mathrm{C}}_{2}-\mathrm{CH}_{2}-\mathrm{CH}_{2}-\underline{\mathrm{C}}_{2}-$ ), 6.88 (d, $1 \mathrm{H}, J \quad 8.3 \mathrm{~Hz}$ ), $6.93(\mathrm{~d}, 1 \mathrm{H}, J 8.3 \mathrm{~Hz}), 6.98(\mathrm{t}, 1 \mathrm{H}, J 7.5 \mathrm{~Hz}), 7.04(\mathrm{t}, 1 \mathrm{H}, J \quad 7.5 \mathrm{~Hz}), 7.29(\mathrm{dt}, 1 \mathrm{H}, J 8.1$ and 1.5 Hz), 7.42 (br. d, 1H, J 7.7 Hz), 7.47 (dt, 1H, J 7.5 and $1.5 \mathrm{~Hz}$ ), 7.64 (d, 1H, J 16.0 Hz, H- $\alpha$ ), 8.08 (dd, $1 \mathrm{H}, J 8.4$ and $1.5 \mathrm{~Hz}$, proton ortho to $\mathrm{CO}$ ), 8.95 (dd, $1 \mathrm{H}, J 15.9$ and $0.8 \mathrm{~Hz}, \mathrm{H}-\beta)$ ) ${ }^{13} \mathrm{C}$ NMR $\left(75 \mathrm{MHz}, \mathrm{CDCl}_{3}\right) \delta_{\mathrm{C}}: 25.93,26.91,68.33,69.07,111.65,112.21,120.55,120.63,124.45$, 127.54, 130.41, 131.66, 131.97, 134.06, 134.34, 138.79, 159.01, 159.42, 190.49 (CO). GCMS: m/z $294\left(\mathrm{M}^{+}\right)$; Anal. Calcd for $\mathrm{C}_{19} \mathrm{H}_{18} \mathrm{O}_{3}$ (294.34): C, 77.53; H, 6.16\%. Found C, 77.28; H, $6.30 \%$.

(2E)-11,17-Dioxatricyclo-[16.4.0.0 $\left.{ }^{5,10}\right]$ docosa-1(22),2,5,7,9,18,20-heptaene-4-one (7c). Eluent: petroleum ether-EtOAc (8:2, v/v), light yellow cubes, mp $112{ }^{\circ} \mathrm{C}$; IR (KBr): 1628 (CO), 1610, 1459, 1301, $1294 \mathrm{~cm}^{-1} ;{ }^{1} \mathrm{H} \mathrm{NMR}\left(300 \mathrm{MHz}, \mathrm{CDCl}_{3}\right) \delta_{\mathrm{H}}: 1.91-2.00\left(\mathrm{~m}, 6 \mathrm{H},-\mathrm{CH}_{2}-\underline{\mathrm{C}}_{2}-\mathrm{C}_{2}-\underline{\mathrm{CH}}_{2}-\right.$ $\left.\mathrm{CH}_{2}-\right), 4.15$ (t, $\left.2 \mathrm{H}, J \quad 4.9 \mathrm{~Hz},-\mathrm{CH}_{2}-\mathrm{CH}_{2}-\mathrm{CH}_{2}-\mathrm{CH}_{2}-\mathrm{CH}_{2}-\right), 4.22$ (t, $2 \mathrm{H}, J \quad 4.9 \mathrm{~Hz},-\mathrm{CH}_{2}-\mathrm{CH}_{2}-\mathrm{CH}_{2}-$ $\left.\mathrm{CH}_{2}-\underline{\mathrm{C}}_{2}{ }^{-}\right), 6.89(\mathrm{~d}, 1 \mathrm{H}, J 8.3 \mathrm{~Hz}), 6.96(\mathrm{t}, 1 \mathrm{H}, J$ 7.4 Hz), $6.99(\mathrm{~d}, 1 \mathrm{H}, J$ 8.3 Hz), $7.01(\mathrm{t}, 1 \mathrm{H}, J$ $7.4 \mathrm{~Hz}), 7.27(\mathrm{dt}, 1 \mathrm{H}, J 7.3$ and $1.5 \mathrm{~Hz}), 7.40-7.44(\mathrm{~m}, 2 \mathrm{H}), 7.68(\mathrm{dd}, 1 \mathrm{H}, J \quad 8.1$ and $1.5 \mathrm{~Hz}$, proton ortho to $\mathrm{CO}), 7.70(\mathrm{~d}, 1 \mathrm{H}, J 15.9 \mathrm{~Hz}, \mathrm{H}-\alpha), 8.20(\mathrm{~d}, 1 \mathrm{H}, J 15.9 \mathrm{~Hz}, \mathrm{H}-\beta) ;{ }^{13} \mathrm{C}$ NMR $(75$ $\left.\mathrm{MHz}, \mathrm{CDCl}_{3}\right) \delta_{\mathrm{C}}: 21.07,25.96,26.58,64.58,68.22,111.60,112.21,120.60,120.70,124.14$, 129.70, 130.17, 130.76, 131.01, 132.82, 134.81, 139.60, 157.53, 159.37, 193.49 (CO); TOFMSES: $m / z 331(\mathrm{M}+\mathrm{Na})^{+}$; Anal. Calcd for $\mathrm{C}_{20} \mathrm{H}_{20} \mathrm{O}_{3}(308.37)$ : C, 77.90; H, 6.54\%. Found C, 78.04; H, 6.78\%.

Macrocyclisation leading to 8a and 9a. The combinations of bis-aldehydes and bis-ketones $\mathbf{1 1 + 2 b}, \mathbf{1 c}+\mathbf{1 2}$ and $\mathbf{1 1 + 1 2}(1: 1$ mole ratio in each case) were treated with aqueous methanolic $\mathrm{KOH}$ in the same way as in the case of condensation of $\mathbf{1}$ and $\mathbf{2}$ described above. Macrocyclization was successful in the first two cases yielding the products 8a and 9a. The analytical and spectral data of these two compounds were as follows: 
$(2 E, 23 E)-11,15,30,34-$ Tetraoxapentacyclo[33.3.1.0 $\left.0^{5,10} \cdot 0^{16,21} \cdot 1^{25,29}\right]$ tetraconta-1(39),2,5,7,9,16,$\mathbf{1 8 , 2 0 , 2 3 , 2 5 , 2 7 , 2 9 ( 4 0 ) , 3 5 , 3 7 - t e t r a d e c a e n e - 4 , 2 2 - d i o n e ~ ( 8 a ) . ~ E l u e n t : ~ p e t r o l e u m ~ e t h e r - E t O A c ~}$ (4:6, v/v), light yellow cubes, mp 206-208 ${ }^{\circ} \mathrm{C}$; IR (KBr): 1635 (CO), 1586, 1489, 1446, $1305 \mathrm{~cm}^{-}$ 1; ${ }^{1} \mathrm{H}$ NMR $\left(300 \mathrm{MHz}, \mathrm{CDCl}_{3}\right) \delta_{\mathrm{H}}: 2.18-2.26\left(\mathrm{~m}, 4 \mathrm{H}, 2 \times-\mathrm{CH}_{2}-\underline{\mathrm{H}}_{2}-\mathrm{CH}_{2}-\right.$ ), 4.10-4.17 (m, 8H, 2 $\left.\times-\mathrm{CH}_{2}-\mathrm{CH}_{2}-\mathrm{CH}_{2}-\right), 6.85-6.91(\mathrm{~m}, 4 \mathrm{H}), 6.94-7.00(\mathrm{~m}, 4 \mathrm{H}), 7.11$ (br. s, 2H), 7.18-7.22 (m, $2 \mathrm{H}), 7.31(\mathrm{~d}, 2 \mathrm{H}, J 15.6 \mathrm{~Hz}, 2 \times \mathrm{H}-\alpha), 7.37(\mathrm{dt}, 2 \mathrm{H}, J 7.5$ and $1.8 \mathrm{~Hz}), 7.54(\mathrm{~d}, 2 \mathrm{H}, J 15.6 \mathrm{~Hz}, 2$ $\times \mathrm{H}-\beta), 7.56-7.59(\mathrm{~m}, 2 \mathrm{H}) ;{ }^{13} \mathrm{C} \mathrm{NMR}\left(75 \mathrm{MHz} \mathrm{CDCl}_{3}\right) \delta_{\mathrm{C}}: 29.02,29.64,64.46,66.08,110.88$, $112.79,117.22$, 121.32, 123.69, 127.10, 129.71, 130.02, 130.53, 133.17, 136.49, 141.65, 157.37, 159.16, $192.49(\mathrm{CO})$; TOFMSES ${ }^{+}: \mathrm{m} / z, 583.49(\mathrm{M}+\mathrm{Na})^{+}$; Anal. Calcd for $\mathrm{C}_{36} \mathrm{H}_{32} \mathrm{O}_{6}(560.22)$ : C, 77.12; H, 5.75\%. Found C, 76.85, H, 5.71\%.

$(2 E, 22 E)-10,15,30,35-T e t r a o x a p e n t a c y c l o\left[34.4 .0 .1^{5,9} \cdot 1^{16,20} .0^{24,29}\right]$ dotetraconta-1(40),2,5,7,-

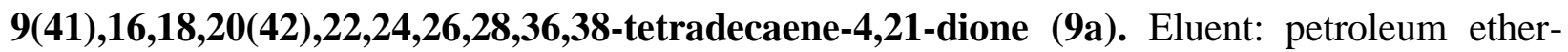
EtOAc (4:6, v/v), light yellow cubes, mp 184-186 ${ }^{\circ} \mathrm{C}$; IR (KBr): 1629 (CO), 1588, 1487, 1446, $1310 \mathrm{~cm}^{-1} ;{ }^{1} \mathrm{H}$ NMR $\left(300 \mathrm{MHz}, \mathrm{CDCl}_{3}\right) \delta_{\mathrm{H}}: 2.03$ (br. s, $4 \mathrm{H}, \mathrm{w}_{1 / 2} \approx 11.5 \mathrm{~Hz},-\mathrm{CH}_{2}-\underline{\mathrm{C}}_{2}-\mathrm{CH}_{2}-\mathrm{CH}_{2}-$ ), 2.17 (br. s, $4 \mathrm{H}, \mathrm{w}_{1 / 2} \approx 11.5 \mathrm{~Hz},-\mathrm{CH}_{2}-\underline{\mathrm{C}}_{2}-\mathrm{C}_{2}-\mathrm{CH}_{2}-$ ), 4.09-4.16 (m, 8H, $2 \times-\underline{\mathrm{C}}_{2}-\mathrm{CH}_{2}-\mathrm{CH}_{2}-$ $\mathrm{C}_{2}-$ ), 6.92 (br. d, 2H, J 8.4 Hz), 7.00 (br. t, 2H, J $7.5 \mathrm{~Hz}$ ), 7.05 (dd, 2H, J 8.1 and $2.2 \mathrm{~Hz}$ ), 7.28-7.57 (m, 10H, Ar-H), 7.66 (d, 2H, J $16.0 \mathrm{~Hz}, 2 \times \mathrm{H}-\alpha), 7.93$ (d, 2H, J $16.0 \mathrm{~Hz}, 2 \times \mathrm{H}-\beta)$; ${ }^{13} \mathrm{C}$ NMR $\left(75 \mathrm{MHz}, \mathrm{CDCl}_{3}\right) \delta_{\mathrm{C}}: 25.45,25.67,66.75,67.44,111.88,114.85,118.50,120.83$, 120.93, 123.90, 124.27, 129.69, 131.22, 131.50, 139.74, 141.44, 158.14, 158.69, 191.71 (CO); TOFMSES ${ }^{+}: m / z 611.53(\mathrm{M}+\mathrm{Na})^{+}$; Anal. Calcd for $\mathrm{C}_{38} \mathrm{H}_{36} \mathrm{O}_{6}(588.25): \mathrm{C}, 77.53 ; \mathrm{H}, 6.16 \%$. Found C, 77.40; H, 6.06\%.

\section{Crystallographic data collection and refinement}

A suitable single crystal of compound $\mathbf{3 f}$ (crystallized from acetone and n-hexane) was mounted on a thin glass fiber with commercially available super glue. X-ray single crystal data collection was performed at room temperature using "Bruker SMART" diffractometer, equipped with a normal focus, sealed tube X-ray source with graphite monochromated Mo-K $\alpha$ radiation $(\lambda$ $0.71073 \AA$ ). The structure was solved by SHELXS 97. Structure refinement was carried out using SHELXL 97. The relevant data are given in Table 3. "CCDC 887597" contains the supplementary crystallographic data for this paper. These data can be obtained free of charge from the Cambridge Crystallographic Data Centre.

Table 3. Important crystallographic data for compound $\mathbf{3 f}$

\begin{tabular}{ll}
\hline Molecular formula & $\mathrm{C}_{36} \mathrm{H}_{32} \mathrm{O}_{6}$ \\
$\mathrm{FW}\left(\mathrm{g} . \mathrm{mol}^{-1}\right)$ & 560.62 \\
Space group & $P 2{ }_{1} / \mathrm{c}$ \\
Temperature & $293{ }^{\circ} \mathrm{K}$ \\
$\mu(\mathrm{Mo}-\mathrm{K} \alpha)$ & $0.085 \mathrm{~mm}^{-1}$ \\
$\mathrm{~F}(000)$ & 1200 \\
\hline
\end{tabular}


Table 3. Continued

\begin{tabular}{ll}
\hline $\mathrm{a} 19.407(3) \mathrm{A}^{\mathrm{o}}$ & $\alpha 90^{\circ}$ \\
$\mathrm{b} 10.6494(17) \mathrm{A}^{\mathrm{o}}$ & $\beta 107.18^{\circ}$ \\
$\mathrm{c} 14.905(2) \mathrm{A}^{\mathrm{o}}$ & $\gamma 90^{\circ}$ \\
Theta Min-Max & $2.20,25.37$ \\
$\mathrm{Vol}\left[\AA^{3}\right]$ & $2943.1(8)$ \\
$\mathrm{Z}$ & 4 \\
$\mathrm{D}$ calc $\left(\mathrm{g} \cdot \mathrm{cm}^{-3}\right)$ & 1.265 \\
Index range & $-22<\mathrm{h}<22$ \\
& $-12<\mathrm{k}<12$ \\
& $-17<1<17$ \\
Number of data measured & 13600 \\
Number of unique data & 4962 \\
Goodness-of-fit & 1.038 \\
Final R indices & $\mathrm{R} 10.0517, w \mathrm{R} 20.1580$ \\
\hline
\end{tabular}

\section{Acknowledgements}

Financial assistance from the UGC-CAS and DST-PURSE programs, Department of Chemistry, Jadavpur University is gratefully acknowledged. The authors also acknowledge the DST-FIST program to the Department of Chemistry, Jadavpur University for the NMR spectral data and the help of Professor A. Ghosh, Head, Department of Chemistry, University of Calcutta for the Xray crystallographic data. One of them (RM) is thankful to the UGC, New Delhi for the award of a research fellowship.

\section{References and Notes}

1. Dhar, D. N.; The Chemistry of Chalcones and Related Compounds, Wiley: New York, 1981.

2. Baviskar, B.; Patel, S.; Baviskar, B.; Khadebadi, S. S.; Shiradkar, M. Asian J. Res. Chem. 2008, $1,67$.

3. Das, B. C.; Marappan, G.; Saha, S.; Bhowmik, D.; Chiranjib, J. Chem. Pharm. Res. 2010, 2, 113.

4. Solankee, A.; Lad, S.; Solankee, S.; Patel, G. Indian J. Chem. 2009, 48B, 1442.

5. Li, R.; Kenyon, G. L.; Cohen, F. E.; Chen, X.; Gong, B.; Dominguez, J. M.; Davidson, E.; Kurzban, G.; Miller, R. E.; Nazum, E. O.; Rosenthal, P. J.; Mckerrow, J. H. J. Med. Chem. 1995, 38, 5031.

6. Solankee, A.; Kapadia, K.; Ćirić, A.; Soković, M.; Doytchinova, I.; Geronikaki, A. Eur. J. Med. Chem. 2010, 45, 510. 
7. Asiri, A. M.; Khan, S. A.; Molbank 2010, M692.

8. Siddiqui, Z. N.; Asad, M.; Praveen, S. Med. Chem. Res. 2008, 17, 318.

9. Trivedi, A. R.; Dodiya, D. K.; Ravat, N. R.; Shah, V. H. Arkivoc 2008, (xi), 131.

10. Voskienè, A.; Mickevičius, V. Chem. Heterocycl. Comp. 2009, 45, 1842.

11. Sandeep, G.; Ranganath, Y. S.; Bhasker, S.; Rajkumar, N. Asian J. Res. Chem. 2009, 2, 46.

12. Vögtle, F. Supramolecular Chemistry, Wiley and Sons: Chichester, 1991.

13. Sauvage, J. P. Ed.; Molecular Machines and Motors, Springer-Verlag: Berlin, 2001.

14. Lehn, J. -M. Supramolecular Chemistry: Concepts and Perspectives, VCH: Weinheim, 1995.

15. Cram, D. J.; Cram, J. M. Container Molecules and Their Guests, Royal Society of Chemistry: Cambridge, 1994.

16. Matsushima, R.; Fujimoto, S.; Tokumura, K. Bull. Chem. Soc. Jpn. 2001, 74, 827.

17. Rurack, K.; Dekhtyhar, M. L.; Bricks, J. L.; Genger, U. R.; Rettig, W. J. Phys. Chem. A 1999, 103, 9626.

18. Vögtle, F.; Mayenfels, P.; Luppertz, F. Synthesis 1984, 580 [Yields of cyclic dichalcones: 5$41 \%$, it was $48-60 \%$ only in one case].

19. Rao, M. L. N.; Hirohiko, H.; Hiratani, K. Tetrahedron Lett. 2001, 42, 8351 [Yields of cyclic dichalcones: $26-41 \%$ ].

20. Cibin, F. R.; Doddi, G.; Mencarelli, P. Tetrahedron 2003, 59, 3455.

21. Higham, L. T.; Kreher, U. P.; Raston, C. L.; Scott, J. L.; Strauss, C. R. Org. Lett. 2004, 6, 3261.

22. Higham, L. T.; Krehar, U. P.; Raston, C. L.; Scott, J. L.; Strauss, C. R. Org. Lett. 2004, 6, 3257.

23. Lakkakula, S.; Mitkin, O. D.; Valiulin, R. A.; Kutateladze, A. G. Org. Lett. 2007, 9, 1077.

24. Ziach, K.; Jurczak, J. Org. Lett. 2008, 10, 5159.

25. Foroughifar, N.; Mobinikhaledi, A.; Ebrahimi, S.; Moghanian, H.; Fard, M. A. B.; Kalhor, M. Tetrahedron Lett. 2009, 50, 836.

26. Avasthi, K.; Ansari, A.; Tiwari, A. K.; Kant, R.; Maulik, P. K. Org. Lett. 2009, 11, 5290.

27. Ito, Y.; Yoshinaga, T.; Nishino, H. Tetrahedron 2010, 66, 2683.

28. Luning, U.; Mak, E.; Zindler, M.; Hartkopf, B.; Herges, R. Eur. J. Org. Chem. 2010, 4932.

29. Singh, P.; Verma, R. K.; Singh, M. S. Tetrahedron Lett. 2011, $52,3818$.

30. Khalil, N. S. A. M. Eur. J. Med. Chem. 2010, 45, 5265.

31. Tirado-Rives, J.; Oliver, M. A.; Fronczek, F. R.; Gandour, R. D. J. Org. Chem. 1984, 49, 1627.

32. Nikitin, K. V.; Andryukhova, N. P. Can. J. Chem. 2004, 82, 571.

33. Yields at different concentrations: (i) concn. $7.5 \times 10^{-3} \mathrm{~mol} / \mathrm{L}: \mathbf{3 a}-48, \mathbf{3 f}-49, \mathbf{3 k}-45, \mathbf{3 p}-40 \%$; (ii) concn. $4.0 \times 10^{-3} \mathrm{~mol} / \mathrm{L}$ : 3a-56, 3f-54, 3k-50, 3p-45\%; (iii) concn. $1.56 \times 10^{-3} \mathrm{~mol} / \mathrm{L}$ : $\mathbf{3 a}-$ 64, 3f-72, 3k-56, 3p-51\%; (iv) concn. $0.8 \times 10^{-3}$ mol/L: 3a-65, 3f-72, 3k-55, 3p-52\%. 